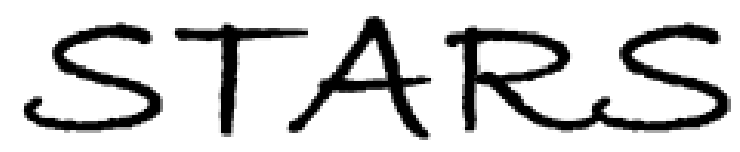

University of Central Florida

STARS

$1-1-2012$

\title{
An Enhanced Hypercube-Based Encoding for Evolving the Placement, Density, and Connectivity of Neurons
}

\author{
Sebastian Risi \\ University of Central Florida \\ Kenneth O. Stanley \\ University of Central Florida
}

Find similar works at: https://stars.library.ucf.edu/facultybib2010 University of Central Florida Libraries http://library.ucf.edu

This Article is brought to you for free and open access by the Faculty Bibliography at STARS. It has been accepted for inclusion in Faculty Bibliography 2010 s by an authorized administrator of STARS. For more information, please contactSTARS@ucf.edu.

\section{Recommended Citation}

Risi, Sebastian and Stanley, Kenneth O., "An Enhanced Hypercube-Based Encoding for Evolving the Placement, Density, and Connectivity of Neurons" (2012). Faculty Bibliography 2010s. 3197.

https://stars.library.ucf.edu/facultybib2010/3197

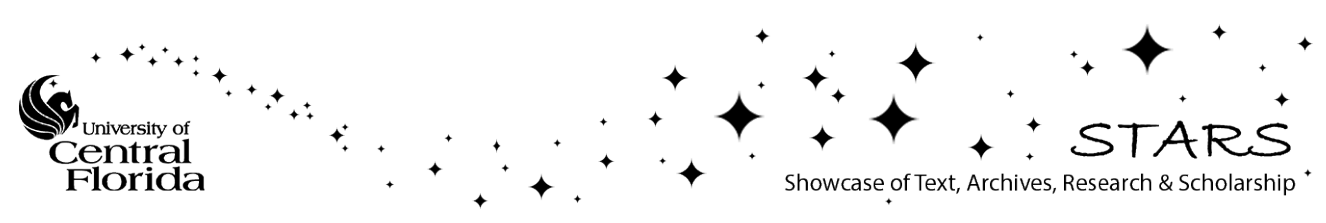




\section{An Enhanced Hypercube-Based Encoding for Evolving the Placement, Density, and Connectivity of Neurons}

\begin{abstract}
Intelligence in nature is the product of living brains, which are themselves the product of natural evolution. Although researchers in the field of neuroevolution (NE) attempt to recapitulate this process, artificial neural networks (ANNs) so far evolved through NE algorithms do not match the distinctive capabilities of biological brains. The recently introduced bypercube-based neuroevolution of augmenting topologies (HyperNEAT) approach narrowed this gap by demonstrating that the pattern of weights across the connectivity of an ANN can be generated as a function of its geometry, thereby allowing large ANNs to be evolved for high-dimensional problems. Yet the positions and number of the neurons connected through this approach must be decided a priori by the user and, unlike in living brains, cannot change during evolution. Evolvable-substrate HyperNEAT (ES-HyperNEAT), introduced in this article, addresses this limitation by automatically deducing the node geometry from implicit information in the pattern of weights encoded by HyperNEAT, thereby avoiding the need to evolve explicit placement. This approach not only can evolve the location of every neuron in the network, but also can represent regions of varying density, which means resolution can increase holistically over evolution. ES-HyperNEAT is demonstrated through multi-task, maze navigation, and modular retina domains, revealing that the $\mathrm{ANNs}$ generated by this new approach assume natural properties such as neural topography and geometric regularity. Also importantly, ES-HyperNEAT's compact indirect encoding can be seeded to begin with a bias toward a desired class of ANN topographies, which facilitates the evolutionary search. The main conclusion is that ES-HyperNEAT significantly expands the scope of neural structures that evolution can discover.
\end{abstract}

Sebastian Risi*,**

University of Central Florida

Kenneth O. Stanley**

University of Central Florida

\begin{abstract}
Keywords
Compositional pattern-producing networks, indirect encoding, HyperNEAT, neuroevolution, artificial neural networks, generative and developmental systems

$A$ version of this paper with color figures is available online at http://dx.doi.ong/10.1162/ artl_a_00071. Subscription required.
\end{abstract}

\section{Introduction}

An ambitious long-term goal for neuroevolution - that is, evolving artificial neural networks (ANNs) through evolutionary algorithms - is to evolve brainlike neurocontrollers with billions of neurons and

\footnotetext{
* Contact author.

** Department of Electrical Engineering and Computer Science, University of Central Florida, Orlando, FL 328I6-2362. E-mail: sebastian.risi@ gmail.com (S.R.); kstanley@cs.ucf.edu (K.O.S.)
} 
trillions of connections. Yet while neuroevolution has produced successful results in a variety of domains $[17,42,54,56,66]$, the scale of natural brains remains far beyond reach. The 100-trillionconnection human brain is fair to describe as the most complex system known to exist [28, 67]. However, its functionality stems not only from the astronomically high number of neurons and connections, but also from its organizational structure, with regularities and repeating motifs such as cortical columns [51].

As evolutionary algorithms are asked to evolve increasingly large and complex structures, interest has increased in recent years in indirect neural network encodings, wherein the description of the solution is compressed in such a way that information can be reused $[2,4-6,14,15,19,22,26,27,30,37$, $39,40,52,57,66]$. Such compression allows the final solution to contain more components than its description. Nevertheless, neuroevolution has historically produced networks with orders of magnitude fewer neurons and significantly less organization and regularity than natural brains $[58,66]$.

While past approaches to neuroevolution generally concentrated on deciding which node is connected to which (i.e., neural topology) $[17,58,66]$, the recently introduced hypercube-based neuroevolution of augmenting topologies (HyperNEAT) method $[11,18,55]$ provided a new perspective on evolving ANNs by showing that the pattern of weights across the connectivity of an ANN can be generated as a function of its geometry. HyperNEAT employs an indirect encoding called compositional pattern-producing networks (CPPNs) [52], which can compactly encode patterns with regularities such as symmetry, repetition, and repetition with variation. In effect, the CPPN in HyperNEAT paints a pattern within a four-dimensional hypercube that is interpreted as the isomorphic connectivity pattern.

HyperNEAT exposed the fact that neuroevolution benefits from neurons that exist at locations within the space of the brain and that by placing neurons at locations, evolution can exploit topography (as opposed to just topology), which makes it possible to correlate the geometry of sensors with the geometry of the brain. While lacking in many ANNs, such geometry is a critical facet of natural brains that is responsible, for example, for topographic maps and modular organization across space [51]. This insight allowed large ANNs with regularities in connectivity to evolve through HyperNEAT for high-dimensional problems $[9,18,19,55]$. Yet a significant limitation is that the positions of the nodes connected through this approach must be decided a priori by the user. In other words, in the original HyperNEAT, the user must explicitly place nodes at locations within a two-dimensional or three-dimensional space called the substrate.

This requirement does not merely create a new task for the user. A more subtle consequence is that if the user dictates that hidden node $n$ must exist at position $(a, b)$ as in the original Hyper-NEAT, it creates the unintentional constraint that any pattern of weights encoded by the CPPN must intersect position $(a, b)$ precisely with the correct weights. That is, the pattern generated by the CPPN in HyperNEAT must perfectly align the correct weights through all points $\left(a, b, x_{2}, y_{2}\right)$ and $\left(x_{1}, y_{1}, a, b\right)$. Yet why should such an arbitrary a priori constraint on the locations of weights be imposed? It might be easier for the CPPN to represent the correct pattern at a slightly different location, yet that would fail under the user-imposed convention.

The key insight in this article is that a representation that encodes the pattern of connectivity across a network (such as in HyperNEAT) automatically contains implicit clues on where the nodes should be placed to best capture the information stored in the connectivity pattern. That is, areas of uniform weight ultimately encode very little information and hence little of functional value. Thus connections (and hence the node locations that they connect) can be chosen to be expressed according to the variance within their region of the $\mathrm{CPPN}$-encoded function in the hypercube from which weights are chosen. In other words, to evolve the locations of nodes, there is no need for any new information or any new representational structure beyond the very same CPPN that already encodes network connectivity in HyperNEAT. Thus this article offers a comprehensive introduction to evolvable-substrate HyperNEAT (ES-HyperNEAT), which was first described in conference papers by Risi et al. [44], where it was introduced, and Risi and Stanley [46], where it was further refined.

The ES-HyperNEAT approach is able to fully determine the internal geometry of node placement and density, based only on implicit information in an infinite-resolution pattern of weights. Thus the 
evolved ANNs exhibit natural properties such as topography and regularity without any need to evolve explicit hidden-node placement. Because the placement of hidden nodes is entirely determined by the algorithm, it circumvents the drawback of the original HyperNEAT that a pattern of weights encoded by the CPPN must intersect specific positions with precisely the correct weights. Also importantly, this enhanced approach has the potential to create networks from several dozen nodes up to several million, which will be necessary in the future to evolve more intelligent systems.

The main conclusion is that ES-HyperNEAT takes a step toward more biologically plausible ANNs and significantly expands the scope of neural structures that evolution can discover, as demonstrated by a series of experiments in this article. The first experiment, in a multi-task domain, explores ES-HyperNEAT's ability to evolve networks with multimodal input. The second experiment, in a deceptive maze navigation domain, shows that ES-HyperNEAT is able to elaborate on an existing structure by holistically increasing the number of synapses and neurons in the ANN during evolution. The third experiment, called the modular left \& right retina problem [8, 29], indicates that ES-HyperNEAT can more easily evolve modular ANNs than the original HyperNEAT, because it has the capability to start the evolutionary search with a bias toward locality and from certain canonical ANN topographies. The idea of seeding with a bias toward certain types of structures is important because it provides a mechanism for emulating key biases in the natural world that are implicitly provided by physics, and it makes it possible to insert specific kinds of domain knowledge into the evolutionary search.

The article begins with a review of NEAT and HyperNEAT in the next section. ES-HyperNEAT is then motivated in Section 3, together with a description of the primary insight. The approach is then detailed in Sections 4 and 5. Next, Sections 6, 7, and 8 present and describe results in the dual task, maze navigation, and retina domains. The article concludes with a discussion and ideas for future work in Section 9.

\section{Background}

This section reviews NEAT and HyperNEAT, which are the foundation of the ES-HyperNEAT approach introduced in this article.

\section{I Neuroevolution of Augmenting Topologies}

The HyperNEAT method that enables learning from geometry is an extension of the original NEAT algorithm that evolves ANNs through a direct encoding.

The NEAT method was originally developed to evolve ANNs to solve difficult control and sequential decision tasks and has proven successful in a wide diversity of domains $[1,53,54,56,60$, 65]. Evolved ANNs control agents that select actions based on their sensory inputs. NEAT is unlike many previous methods that evolved neural networks (i.e., neuroevolution methods), which traditionally evolve either fixed-topology networks $[20,48]$ or arbitrary random-topology networks $[3,22$, 66]. Instead, NEAT begins evolution with a population of small, simple networks and complexifies the network topology into diverse species over generations, leading to increasingly sophisticated behavior. A similar process of gradually adding new genes has been confirmed in natural evolution [36, 64] and shown to improve adaptation in a few prior evolutionary [64] and neuroevolutionary [25] approaches. However, a key feature that distinguishes NEAT from prior work in complexification is its unique approach to maintaining a healthy diversity of complexifying structures simultaneously, as this section reviews. Complete descriptions of the NEAT method, including experiments confirming the contributions of its components, are available in Stanley and Miikkulainen [56, 58] and Stanley et al. [54].

The NEAT method is based on three key ideas. First, to allow network structures to increase in complexity over generations, a method is needed to keep track of which gene is which. Otherwise, it is not clear in later generations which individual is compatible with which in a population of diverse structures, or how their genes should be combined to produce offspring. NEAT solves this problem by assigning a unique historical marking to every new piece of network structure that appears through a structural mutation. The historical marking is a number assigned to each gene corresponding to its 
order of appearance over the course of evolution. The numbers are inherited during crossover unchanged, and allow NEAT to perform crossover among diverse topologies without the need for expensive topological analysis.

Second, historical markings make it possible for the system to divide the population into species based on how similar networks are topologically. That way, individuals compete primarily within their own niches instead of with the population at large. Because adding new structure is often initially disadvantageous, this separation means that unique topological innovations are protected and therefore have time to optimize their structure before competing with other niches in the population.

Third, many systems that evolve network topologies and weights begin evolution with a population of random topologies [22, 66]. In contrast, NEAT begins with a uniform population of simple networks with no hidden nodes, differing only in their initial random weights. Because of speciation, novel topologies gradually accumulate over evolution, thereby allowing diverse and complex phenotype patterns to be represented. No limit is placed on the size to which topologies can grow. New structure is introduced incrementally as structural mutations occur, and only those structures survive that are found to be useful through fitness evaluations. In effect, then, NEAT searches for a compact, appropriate topology by incrementally increasing the complexity of existing structure.

The next section reviews generative and developmental systems (GDSs), focusing on compositional patternproducing networks (CPPNs) and the HyperNEAT approach, which will be extended in this article.

\subsection{Generative and Developmental Systems}

In direct encodings like NEAT, each part of the solution's representation maps to a single piece of structure in the final solution $[17,66]$. The significant disadvantage of this approach is that even when different parts of the solution are similar, they must be encoded and therefore discovered separately. Thus this article employs an indirect encoding instead, which means that the description of the solution is compressed in such a way that information can be reused, allowing the final solution to contain more components than the description itself. Indirect encodings, which are the focus of the field of GDSs, are powerful because they allow solutions to be represented as a pattern of parameters, rather than requiring each parameter to be represented individually $[5,6,19,24,26$, $38,52,57]$. The next section reviews one such indirect encoding in more detail.

\subsection{Compositional Pattern-Producing Networks}

Recently, NEAT was extended to evolve a high-level developmental abstraction called compositional pattern-producing networks (CPPNs) [52]. The idea behind CPPNs is that patterns in nature can be described at a high level as compositions of functions, wherein each function represents a stage in development. CPPNs are similar to ANNs, but they rely on more than one activation function (each representing a common regularity). Interestingly, because CPPNs are also connected graphs, they can be evolved by NEAT just like ANNs. Thus the CPPN encoding does not require a new evolutionary algorithm to evolve.

The indirect CPPN encoding can compactly encode patterns with regularities such as symmetry, repetition, and repetition with variation $[49,50,52]$. For example, simply by including a Gaussian function, which is symmetric, the output pattern can become symmetric. A periodic function such as the sine creates segmentation through repetition. Most importantly, repetition with variation (e.g., in the fingers of the human hand) is easily discovered by combining regular coordinate frames (e.g., sine and Gaussian) with irregular ones (e.g., the asymmetric $x$ axis). For example, a function that takes as input the sum of a symmetric function and an asymmetric function outputs a pattern with imperfect symmetry. In this way, CPPNs produce regular patterns with subtle variations. The potential for CPPNs to represent patterns with motifs reminiscent of patterns in natural organisms has been demonstrated in several studies [49, 50, 52]. Specifically, CPPNs produce a phenotype that is a function of $n$ dimensions, where $n$ is the number of dimensions in physical space. For each coordinate in that space, its level of expression is an output of the function that encodes the phenotype. Figure 1 shows how a two-dimensional phenotype can be generated by a function of two parameters that is represented by a network of 


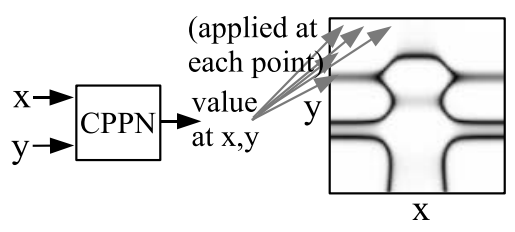

(a) Mapping

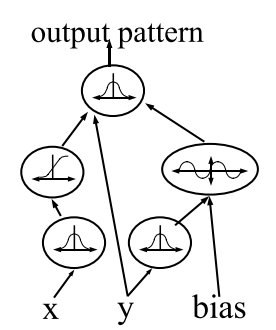

(b) Composition

Figure I. CPPN encoding. (a) The function $f$ takes arguments $x$ and $y$, which are coordinates in a two-dimensional space. When all the coordinates are drawn with an intensity corresponding to the output of $f$, the result is a spatial pattern, which can be viewed as a phenotype whose genotype is $f$. (b) The CPPN is a graph that determines which functions are connected. The connections are weighted so that the output of a function is multiplied by the weight of its outgoing connection.

composed functions. Because CPPNs are a superset of traditional ANNs, which can approximate any function [10], CPPNs are also universal function approximators. Thus a CPPN can encode any pattern within its $n$-dimensional space. The next section reviews the HyperNEAT extension to NEAT, which is itself extended in this article.

\subsubsection{HyperNEAT}

HyperNEAT, reviewed in this section, is an indirect encoding extension of NEAT that is proven in a number of challenging domains that require discovering regularities [8, 12, 18, 19, 55, 61]. For a full description of HyperNEAT see Stanley et al. [55] and Gauci and Stanley [19].

The main idea in HyperNEAT is to extend CPPNs, which encode spatial patterns, to also represent connectivity patterns $[7,18,19,55]$. That way, NEAT can evolve CPPNs that represent large-scale ANNs with their own symmetries and regularities. The key insight is that $2 n$-dimensional spatial patterns are isomorphic to connectivity patterns in $n$ dimensions, that is, patterns in which the coordinate of each endpoint is specified by $n$ parameters. Consider a CPPN that takes four inputs labeled $x 1$, $y 1, x 2$, and $y 2$; this point in four-dimensional space also denotes the connection between the twodimensional points $(x 1, y 1)$ and $(x 2, y 2)$, and the output of the CPPN for that input thereby represents the weight of that connection (Figure 2). By querying every possible connection among a set of points in this manner, a CPPN can produce a neural network, wherein each queried point is a neuron position. The space in which these neurons are positioned is called the substrate. Because the connections are produced by a function of their endpoints, the final structure is produced with knowledge of

1) Query each potential connection on substrate

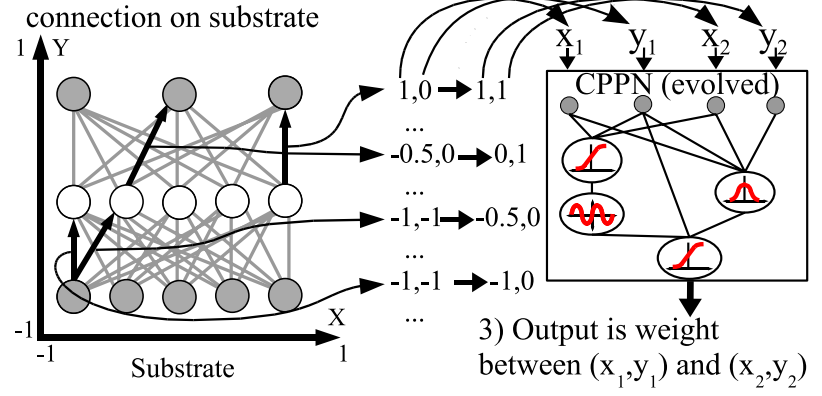

Figure 2. Interpretation of hypercube-based geometric connectivity pattern. A collection of nodes, called the substrate, is assigned coordinates that range from $-I$ to $I$ in all dimensions. (I) Every potential connection in the substrate is queried to determine its presence and weight; the dark directed lines in the substrate depicted in the figure represent a sample of connections that are queried. (2) Internally, the CPPN (which is evolved) is a graph that determines which activation functions are connected. As in an ANN, the connections are weighted so that the output of a function is multiplied by the weight of its outgoing connection. For each query, the CPPN takes as input the positions of the two endpoints and (3) outputs the weight of the connection between them. Thus, CPPNs can produce regular patterns of connections in space. 
its geometry. In effect, the CPPN is painting a pattern on the inside of a four-dimensional hypercube that is interpreted as an isomorphic connectivity pattern, which explains the origin of the name Hypercube-based NEAT (HyperNEAT). Connectivity patterns produced by a CPPN in this way are called substrates so that they can be verbally distinguished from the CPPN itself, which has its own internal topology.

Each queried point in the substrate is a node in an ANN. In traditional implementations of HyperNEAT the experimenter specifies both the location and role (i.e., hidden, input, or output) of each such node. As a rule of thumb, nodes are placed on the substrate to reflect the geometry of the task $[8,12,18,55]$. That way, the connectivity of the substrate is a function of the task structure.

For example, the sensors of an autonomous robot can be placed from left to right on the substrate in the same order as on the robot. Outputs for moving left or right can also be placed in the same order, allowing HyperNEAT to understand from the outset the correlation of sensors to effectors. In this way, knowledge about the problem geometry can be injected into the search, and HyperNEAT can exploit the regularities (e.g., adjacency, or symmetry) of a problem that are invisible to traditional encodings.

The conventional method for controlling connectivity in HyperNEAT is a threshold that limits the range of values output by the CPPN that can be expressed as weights. The threshold is a parameter specified at initialization that is uniformly applied to all connections queried. When the magnitude of the output of the CPPN is below this threshold, the connection is not expressed.

However, Verbancsics and Stanley [62] introduced an alternative to the traditional uniform threshold, called the link expression output (HyperNEAT-LEO), that allowed HyperNEAT to evolve the pattern of weights independently from the pattern of connection expression. The LEO is represented as an additional output to the CPPN that indicates whether a connection should be expressed or not. If the LEO output is greater than zero, then the corresponding connection is created and its weight is set to the original CPPN weight output value. Because HyperNEAT evolves such patterns as functions of geometry, important general topographic principles for organizing connectivity can be seeded into the initial population [62]. For example, HyperNEAT can be seeded with a bias toward local connectivity implemented through LEO, in which locality is expressed through a Gaussian function. Because the Gaussian function peaks when its input is 0.0 , inputting a difference between coordinates (e.g., $\Delta x$ ) achieves the highest value when the coordinates are the same. In this way, such seeds provide the concept of locality, because the more local the connection (i.e., as $\Delta x$ approaches 0.0 ), the greater the output of the Gaussian function.

Because there are now two different thresholding methods for HyperNEAT, the new approach introduced in this article is compared with both. However, regardless of the approach to thresholding, a problem that has endured with HyperNEAT is that the experimenter is left to decide how many hidden nodes there should be and where to place them too. That is, although the CPPN determines how to connect nodes in a geometric space, it does not specify where the nodes should be, which is especially ambiguous for hidden nodes.

In answer to this challenge, the next section introduces an extension to HyperNEAT in which the placement and density of the hidden nodes do not need to be set a priori and in fact are completely determined by implicit information in the CPPN itself.

\section{Choosing Connections to Express}

The placement of nodes in original HyperNEAT is decided by the user. Yet whereas it is often possible to determine how sensors and effectors relate to domain geometry, it is difficult for the user to determine the best placement and number of necessary hidden nodes a priori. For example, the location of the hidden nodes in the substrate in Figure 2 had to be decided by the user. HyperNEAT thus creates the strange situation that it can decide with what weight any two nodes in space should be connected, but it cannot tell us anything about where the nodes should be. Is there a representation that can evolve the placement and density of nodes and that can potentially span the range between networks of several dozen nodes and several billion? 


\section{I Implicit Information in the Hypercube}

The novel insight behind ES-HyperNEAT is that a representation that encodes the pattern of connectivity across a network automatically contains implicit information that could be useful for deciding where the nodes should be placed. In HyperNEAT the pattern of connectivity is described by the CPPN, where every point in the four-dimensional space denotes a potential connection between two two-dimensional points (recall that a point in the four-dimensional hypercube is actually a connection weight and not a node). Because the CPPN takes $x_{1}, y_{1}, x_{2}$, and $y_{2}$ as input, it is a function of the infinite continuum of possible coordinates for these points. In other words, the CPPN encodes a potentially infinite number of connection weights within the hypercube of weights. Thus one interesting way to think about the hypercube is as a theoretically infinite pattern of possible connections that might be incorporated into a neural network substrate. If a connection is chosen to be included, then by necessity the nodes that it connects must also be included in the substrate. Thus by asking which connections to include from the infinite set, we are also asking which nodes (and hence their positions) to include.

By shifting the question of what to include in the substrate from nodes to connections, two important insights follow: First, the more such connections are included, the more nodes would also be added to the substrate. Thus the node density increases with the number of connections. Second, for any given infinite-resolution pattern, there is some sampling density above which increasing the density further offers no advantage. For example, if the hypercube is a uniform gradient of maximal connection weights (i.e., all weights are the same constant), then in effect it encodes a substrate that computes the same function at every node. Thus adding more such connections and nodes adds no new information. On the other hand, if there is a stripe of differing weights running through the hypercube, but otherwise uniform maximal connections everywhere else, then that stripe contains information that would contribute to a different function from its redundantly uniform neighbors.

The key insight is thus that it is not always a good idea to add more connections, because for any given finite pattern, at some resolution there is no more information and adding more weights at such high resolution would be redundant and unnecessary. This maximal useful resolution varies for different regions of the hypercube, depending on the complexity of the underlying weight pattern in those regions. Thus the answer to the question of which connections should be included in ES-HyperNEAT is that connections should be included at high enough resolution to capture the detail (i.e., information) in the hypercube. Any more than that would be redundant. Therefore, an algorithm is needed that can choose many points to express in regions of high variance and fewer points to express in regions of relative homogeneity. Each such point is a connection weight in the substrate, whose respective nodes will be expressed as well. The main principle is simple: Density follows information. In this way, the placement of nodes in the topographic layout of an ANN is ultimately a signification of where information is stored within weights.

To perform the task of choosing points (i.e., weights) to express, a data structure is needed that allows space to be represented at variable levels of granularity. One such multi-resolution technique is the quadtree [16], which traditionally describes two-dimensional regions. It has been applied successfully in fields ranging from pattern recognition to image encoding $[47,59]$ and is based on recursively splitting a two-dimensional region into four subregions. That way, the decomposition of a region into four new regions can be represented as a subtree whose parent is the original region with one descendant for each decomposed region. The recursive splitting of regions can be repeated until the desired resolution is reached or until no further subdivision is needed because additional resolution is no longer uncovering any new information. The next sections describe the ES-HyperNEAT algorithm in more detail. A pseudocode implementation can be found in Appendix 2.

\section{Quadtree Information Extraction}

Instead of searching directly in the four-dimensional hypercube space (recall that it takes four dimensions to represent a two-dimensional connectivity pattern), ES-HyperNEAT iteratively discovers the ANN connections starting from the inputs and outputs of the ANN (which are prespecified by the 
user). This approach focuses the search within the hypercube on two-dimensional cross sections of the hypercube.

The foundation of the ES-HyperNEAT algorithm is the quadtree information extraction procedure, which receives a two-dimensional position as input and then analyzes either the outgoing connectivity pattern from that single neuron (if it is an input), or the incoming connectivity pattern (if it is an output). For example, given an input neuron at $(a, b)$, the quadtree connection-choosing algorithm is applied only to the two-dimensional outgoing connectivity patterns described by the function CPPN $\left(a, b, x_{2}, y_{2}\right)$, where $x_{2}$ and $y_{2}$ range between -1 and 1 . The algorithm works in two main phases (Figure 3): In the division and initialization phase (Figure 3 top) the quadtree is created by recursively subdividing the initial square (lines 8-11 of Algorithm 1 in Appendix 2), which spans the space from $(-1,-1)$ to $(1,1)$, until a desired initial resolution $r$ is reached (e.g., $4 \times 4$, which corresponds to a quadtree depth of 3 ). For every quadtree square with center $(x, y)$, the CPPN is queried with arguments $(a, b, x, y)$ and the resulting connection weight value $w$ is stored (line 14 of Algorithm 1).

Given the values $\left(w_{1}, w_{2}, \ldots, w_{k}\right)$ of the $k$ leaf nodes in a subtree of quadtree node $p$ and mean weight $\bar{w}$, the variance of node $p$ in the quadtree can be calculated as $\sigma_{p}^{2}=\frac{1}{k} \sum_{1}^{k}\left(\bar{w}-w_{i}\right)^{2}$. This variance is a heuristic indicator of the heterogeneity (i.e., presence of information) of a region. If the variance of the parent of a quadtree leaf is still higher than a given division threshold $d_{t}$ (line 20 of Algorithm 1), then the division phase can be reapplied for the corresponding leaf's square, allowing increasingly high densities. Just as the initialization resolution ensures that some minimum level of sampling is enforced (so that the basic shape of the encoded pattern is likely to be discovered), a maximum resolution level $r_{m}$ can also be set to place an upper bound on the number of possible neurons if desired. However, it is theoretically interesting that in principle this algorithm can yield arbitrarily high density, which means that very large ANNs can be represented.

The quadtree representation created in the initialization phase serves as a heuristic variance indicator to decide on the connections (and therefore placement and density of neurons) to express. Because more connections should be expressed in regions of higher variance, a pruning and extraction phase (Algorithm 2 in Appendix 2) is next executed (Figure 3 bottom), in which the quadtree is traversed depth-first until the current node's variance is smaller than the variance threshold $\sigma_{t}^{2}$

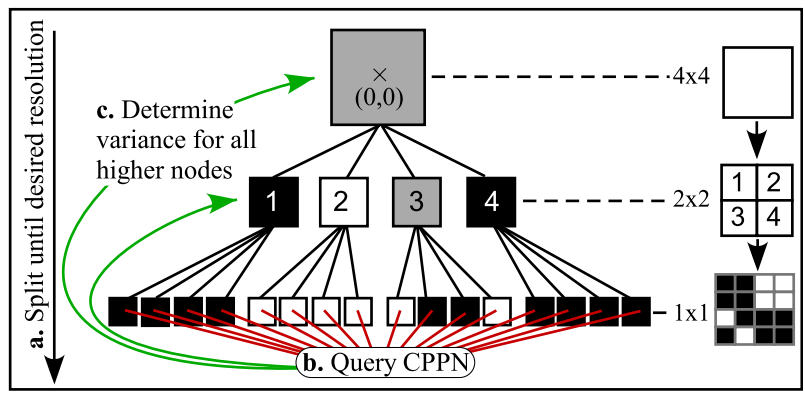

(1) Division And Initialization Phase

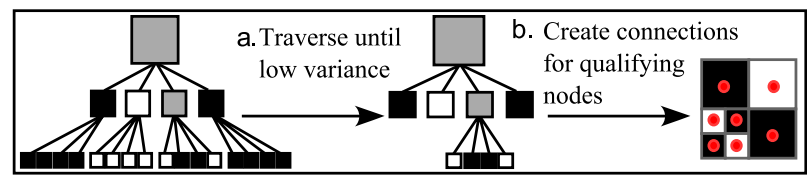

(2) Pruning And Extraction Phase

Figure 3. Quadtree information extraction example. Given an input neuron at $(a, b)$, the algorithm works in two main stages. (I) In the division and initialization phase the quadtree is created by recursively splitting each square into four new squares until the desired resolution is reached $(\mathrm{la})$, while the values $(\mathrm{Ib})$ for each square with center $(x, y)$ are determined by $\operatorname{CPPN}(a, b, x, y)$ and the variance values of each higher node are calculated (Ic). Gray nodes in the figure have a variance greater than zero. Then, in the pruning and extraction phase (2), the quadtree is traversed depth-first until the node's variance is smaller than a given threshold (2a). A connection $(a, b, x, y)$ is created for each qualifying node with center $(x, y)(2 b)$. That way, the density of neurons in different regions will correspond to the amount of information in that region. 


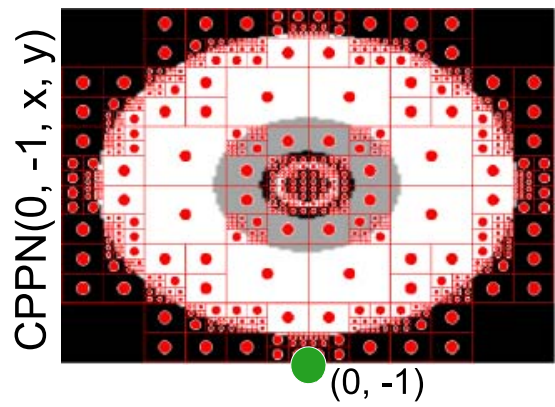

(a) Without band pruning

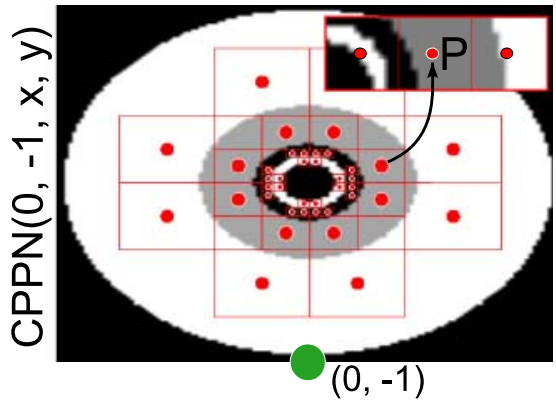

(b) With band pruning

Figure 4. Example connection selection. Chosen connections (depicted only by their target locations) originating from $(0,-I)$ are shown in (a) after the pruning stage but without band pruning. Points that still remain after band pruning (e.g., point $P$, whose neighbors at the same resolution have different CPPN activation levels) are shown in (b). The resulting point distribution reflects the information inherent in the pattern.

(line 4 of Algorithm 2) or until the node has no children (which means that the variance is zero). Subsequently, a connection $(a, b, x, y)$ is created for each qualifying node with center $(x, y)$ (line 22 of Algorithm 2; the band threshold in Algorithm 2 is explained shortly). The result is higher resolution in areas of more variation.

Figure $4 \mathrm{a}$ shows an example of the outgoing connections from the source neuron $(0,-1)$ (depicted only by their target locations for clarity) chosen at this stage of the algorithm. The variance is high at the borders of the circles, which results in a high density of expressed points near those locations. However, for the purpose of identifying connections to include in a neural topography, the raw pattern output by the quadtree algorithm can be improved further. If we think of the pattern output by the $\mathrm{CPPN}$ as a kind of language for specifying the locations of expressed connections, then it makes sense additionally to prune the points around borders so that it is easy for the CPPN to encode points definitively within one region or another.

Thus a more parsimonious language for describing density patterns would ignore the edges and focus on the inner region of bands, which are points that are enclosed by at least two neighbors on opposite sides (e.g., left and right) with different CPPN activation levels (Figure 4b). Furthermore, narrower bands can be interpreted as requests for more point density, giving the CPPN an explicit mechanism for affecting density. Thus, to facilitate banding, a pruning stage is added that removes points that are not in a band. Membership in a band for a square with center $(x, y)$ and width $\omega$ is determined by the band level

$\beta=\max \left(\min \left(d_{\text {top }}, d_{\text {bottom }}\right), \min \left(d_{\text {left }}, d_{\text {right }}\right)\right)$,

where $d_{\text {left }}$ is the difference in CPPN activation levels between the connection $(a, b, x, y)$ and its left neighbor at $(a, b, x-\omega, y)$ (line 9 of Algorithm 2). The other values, $d_{\text {right }}, d_{\text {bottom, and }} d_{\text {top }}$, are calculated similarly. If the band level $\beta$ is below a given threshold $\beta_{t}$, then the corresponding connection is not expressed (line 19 of Algorithm 2). Figure 4b shows the resulting point selections with band pruning.

This approach also naturally enables the CPPN to increase the density of points chosen, by creating more bands or making them thinner. Thus no new information and no new representational structure beyond the CPPN already employed in HyperNEAT is needed to encode node placement and connectivity, as concluded in the next section.

\section{ES-HyperNEAT Algorithm}

The complete ES-HyperNEAT algorithm (Algorithm 3 in Appendix 2) is depicted in Figure 5. The connections originating from an input at $(0,-1)$ (Figure 5a; line 7 in Algorithm 3) are chosen with the approach 
described in the previous section. The corresponding hidden nodes are created if not already existent (lines 9-10 in Algorithm 3). The approach can be iteratively applied to the discovered hidden nodes until a user-defined maximum iteration level is reached (line 15 in Algorithm 3) or no more information is discovered in the hypercube (Figure 5b). To tie the network into the outputs, the approach then chooses connections based on each output's incoming connectivity patterns (Figure 5c; line 27 in Algorithm 3).

Once all hidden neurons are discovered, only those are kept that have a path to an input and an output neuron (Figure 5d; line 34 in Algorithm 3). This iterated approach helps to reduce computational costs by focusing the search on a sequence of two-dimensional cross sections of the hypercube instead of searching for information directly in the full four-dimensional hyperspace.

ES-HyperNEAT ultimately unifies a set of algorithmic advances stretching back to NEAT, each abstracted from an important facet of natural evolution that contributes to its ability to evolve complexity. The first is that evolving complexity requires a mechanism to increase the information content in the genome over generations $[56,58]$. Second, geometry plays an important role in natural neural connectivity; in neuroevolution, endowing neurons with geometric coordinates means that the genome can in effect project regularities in connectivity across the neural geometry, thereby providing a kind of scaffolding for situating cognitive structures $[18,19,55]$. Third, the placement and density of neurons throughout the geometry of the network should reflect the complexity of the underlying functionality of its respective parts [46].

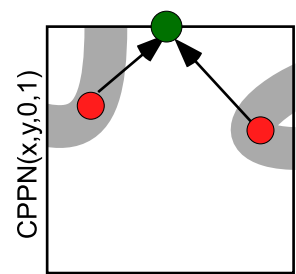

\section{c. Hidden to Output}

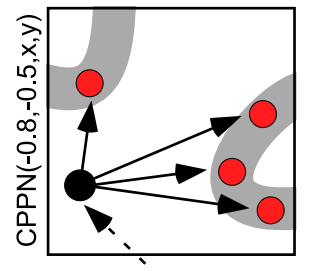

b. Hidden.to Hidden
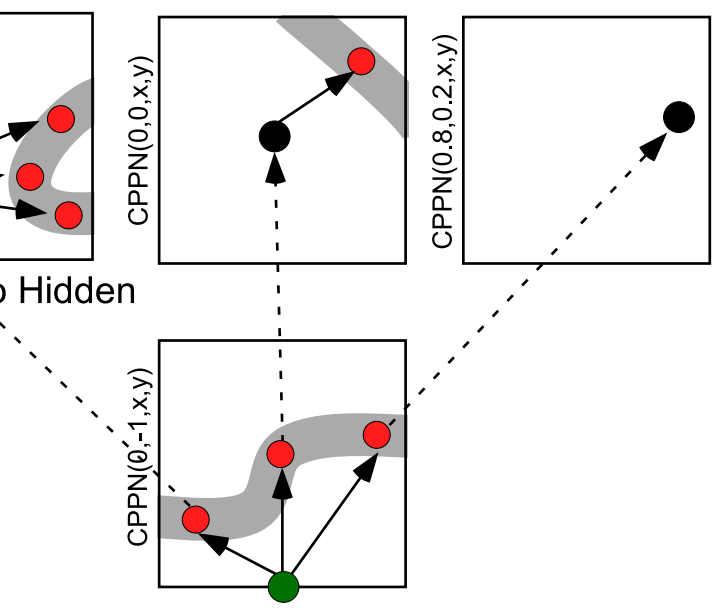

d. Complete Paths

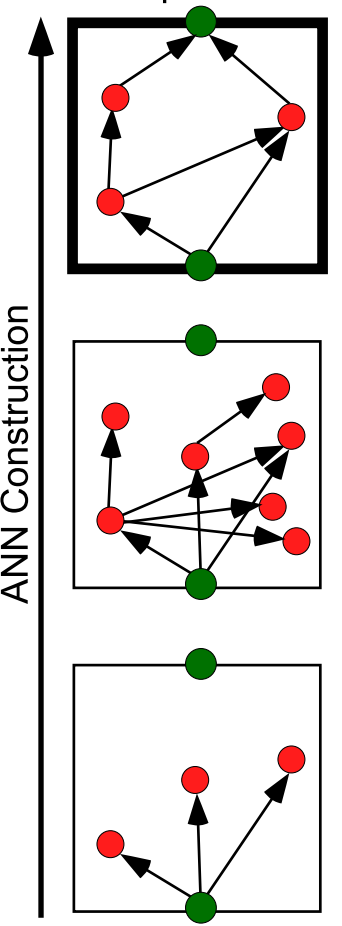

\section{a. Input to Hidden}

Figure 5. The ES-HyperNEAT algorithm. The algorithm starts by iteratively discovering the placement of the hidden neurons from the inputs (a) and then ties the network into the outputs (c). The two-dimensional motif in (a) represents outgoing connectivity patterns from a single input node, whereas the motif in (c) represents incoming connectivity patterns for a single output node. The target nodes discovered (through the quadtree algorithm) are those that reside within bands in the hypercube. In this way regions of high variance are sought only in the two-dimensional cross section of the hypercube containing the source or target node. The algorithm can be iteratively applied beyond the inputs to the discovered hidden nodes (b). Only those nodes are kept at the end that have a path to an input and an output neuron (d). That way, the search through the hypercube is restricted to functional ANN topologies. 
Because ES-HyperNEAT can automatically deduce node geometry and density from CPPNs instead of requiring a priori placement (as in original HyperNEAT), it significantly expands the scope of neural structures that evolution can discover. The approach not only evolves the location of every neuron in the brain, but also can represent regions of varying density, which means resolution can increase holistically over evolution. The main insight is that the connectivity and hidden-node placement can be automatically determined by information already inherent in the pattern encoded by the CPPN. In this way, the density of nodes is automatically determined and effectively unbounded. Thus substrates of unbounded density can be evolved and determined without any additional representation beyond the original CPPN in HyperNEAT.

\section{I Key Hypotheses}

Automatically determining the placement and density of hidden neurons introduces several advantages beyond just liberating the user from making such decisions. This subsection introduces the key hypotheses in this article that elucidate these advantages, which the experiments in Sections 6, 7, and 8 aim to validate.

Hypothesis 1. ES-HyperNEAT facilitates evolving networks with targeted connectivity for multimodal tasks.

Although it produces regular patterns of weights, the original HyperNEAT tends to produce fully or near fully connected networks [8], which may create a disadvantage in domains where certain neurons should only receive input from one modality while other neurons should receive inputs from multiple modalities, thus allowing the sharing of information about the underlying task similarities in the hidden layer. In contrast, because ES-HyperNEAT only creates connections where there is high variance in the hypercube, it should be able to find greater variation in connectivity for different neurons. To test Hypothesis 1, the first experiment explores how ES-HyperNEAT performs in a multitask domain (Section 6), which requires the agent to react differently according to the type of input (e.g., rangefinder or radar) it receives.

Hypothesis 2. The fixed locations of hidden nodes in original HyperNEAT that are chosen by the user make finding an effective pattern of weights more difficult than does allowing the algorithm itself to determine their locations, as in ES-HyperNEAT.

The problem is that when node locations are fixed, the pattern in the hypercube that is encoded by the CPPN must intersect those node coordinates at precisely the right locations. Even if such a CPPN encodes a pattern of weights that expresses an effective network, a slight shift (i.e., a small translation) of the pattern would cause it to detach from the correct node locations. Thus the network would receive a low fitness even though it actually would encode the right pattern if only the nodes were slightly shifted. In contrast, ES-HyperNEAT in effect tracks shifts in the underlying pattern, because the quadtree algorithm searches for the appropriate locations of nodes regardless of exactly where the pattern is expressed. This increased flexibility means that the feasible area of the search space will be larger and hence easier to hit. Analyzing the resulting ANNs will demonstrate whether ES-HyperNEAT can express hidden nodes at slightly different locations when the pattern of weights changes.

Hypothesis 3. ES-HyperNEAT is able to elaborate on existing structure by increasing the number of synapses and neurons in the ANN during evolution, while regular HyperNEAT takes the entire set of ANN connection weights to represent a partial solution.

The second experiment in a deceptive maze navigation domain (Section 7) will isolate this issue by examining the effect of a task with several intermediate milestones on both variants. 
Hypothesis 4. ES-HyperNEAT can evolve modular ANNs more easily than the original fixedsubstrate HyperNEAT, in part because it facilitates evolving networks with limited connectivity and because it has the capability to start the evolutionary search with a bias toward locality and toward certain canonical ANN topographies.

The third experiment, called the left \& right retina problem [7, 29] (Section 8), will test the ability to evolve modular structures, because the task benefits from separating different functional structures. Whereas the original HyperNEAT was extended to allow seeding with a bias toward local connectivity through HyperNEAT-LEO [62], ES-HyperNEAT can also be seeded with a CPPN that creates certain ANN topographies (i.e., geometry seeding). This advance is enabled by ES-HyperNEAT's ability to place the hidden nodes according to the underlying information in the hypercube.

If these hypotheses are correct, then ES-HyperNEAT should not only match but outperform the original HyperNEAT, as the experiments in the next sections will test.

\section{Experiment I: Dual Task}

Organisms in nature have the ability to switch rapidly between different tasks, depending on the demands of the environment. For example, a rat should react differently when placed in a maze or in an open environment with a visible food source. The dual task domain presented here (Figure 6) will test the ability of ES-HyperNEAT and regular HyperNEAT to evolve such task differentiation for a multimodal domain.

The dual task domain consists of two nondependent scenarios (i.e., the performance in one scenario does not directly influence the performance in the other scenario) that require the agent to exhibit different behaviors and to react either to its rangefinders or to its pie-slice sensors. Because certain hidden neurons ideally would be responsible for information that should be treated differently, while other hidden neurons should be able to share information where the tasks are similar, this domain will likely benefit from ANNs that are not fully connected, which the original HyperNEAT has struggled to produce in the past [7]. ES-HyperNEAT should facilitate the evolution of networks with more targeted connectivity, as suggested by Hypothesis 1, because connections are only included at a high enough resolution to capture the information in the hypercube.

The first scenario is a simple navigation task in which the agent has to navigate from a starting point to an end point in a fixed amount of time, using only its rangefinder sensors to detect walls (Figure 6a). The fitness in this scenario is calculated as $f_{\text {nav }}=1-d_{g}$, where $d_{g}$ is the distance of the robot to the goal point at the end of the evaluation, scaled into the range $[0,1]$. The second scenario is a food-gathering task in which a single piece of food is placed within a square room with an agent that begins at the center (Figure 6b). The agent attempts to gather as much food as possible within a time limit, using only its pie-slice sensors, which act as a compass toward the food item. Food only appears at one location at a time and is placed at another random location once consumed by the agent. The fitness for the food-gathering task is defined by $f_{\text {food }}=\frac{n+\left(1-d_{f}\right)}{4}$, where $n$ corresponds to

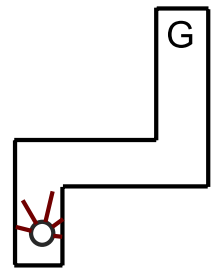

(a) Scenario 1

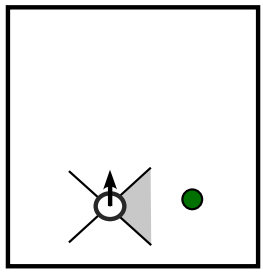

(b) Scenario 2

Figure 6. Dual task. In the dual task domain the agent has to exhibit either wall-following (a) or food-gathering behavior (b), depending on the type of sensory input it receives. 


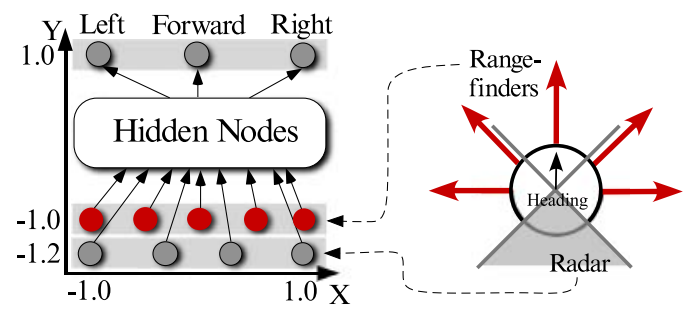

Figure 7. Substrate configuration and sensor layout. The controller substrate is shown at left. Whereas the number of hidden nodes for the fixed-substrate approach is determined in advance, ES-HyperNEAT decides on the positions and density of hidden nodes on its own. The sensor layout is shown on the right. The autonomous agent is equipped with five distance and four pie-slice sensors. Each rangefinder sensor indicates the distance to the closest obstacle in that direction. The pie-slice sensors act as a compass toward the goal (i.e., food), activating when a line from the goal to the center of the robot falls within the pie slice.

the number of collected food items (maximum four), and $d_{f}$ is the distance of the robot to the next food item at the end of the evaluation.

The total fitness is calculated as the average of the fitness values in the two scenarios. The domain is considered solved when the agent is able to navigate to the goal point in the first scenario and successfully collects all four food items in the second scenario, which corresponds to a fitness of 1.0.

\section{I Experimental Setup}

Evolvable and fixed-substrate (original) HyperNEAT use the same placement of input and output nodes on the substrate (Figure 7), which are designed to correlate senses and outputs geometrically (e.g., seeing something on the left and turning left). Thus the CPPN can exploit the geometry of the agent. The agent is equipped with five rangefinder sensors that detect walls and four pie-slice sensors that act as a compass toward the next food item. All rangefinder sensor values are scaled into the range $[0,1]$, where lower activation indicates closer proximity to a wall. A pie-slice sensor is set to 1.0 when a line from the next food item to the center of the robot falls within the pie slice, and is set to 0.0 otherwise. At each discrete moment of time, the number of units moved by the agent is $20 F$, where $F$ is the forward effector output. The agent also turns by $(L-R) \times 18^{\circ}$, where $L$ is the left effector output and $R$ is the right effector output. A negative value is interpreted as a right turn.

To highlight the challenge of deciding the location and number of available hidden nodes, ES-HyperNEAT is compared with four fixed-substrate variants (Figure 8). FS10x1 is the typical setup with a single row of 10 hidden neurons in a horizontal line at $y=0$ (Figure 8a). For the FS1x10 variant 10 hidden neurons are arranged vertically at $x=0$ (Figure $8 \mathrm{~b}$ ). FS5x5 has a substrate containing 25 hidden nodes arranged in a $5 \times 5$ grid (Figure 8c). FS8x8 tests the effects on performance of uniformly increasing the number of hidden nodes from 25 to 64 neurons (Figure 8d). To generate such a controller for the original HyperNEAT, a four-dimensional CPPN with inputs $x_{1}$, $y_{1}, x_{2}$, and $y_{2}$ queries the substrate shown in Figure 7 to determine the connection weights between the input and hidden, the hidden and output, and the hidden and hidden nodes. In contrast, ES-HyperNEAT decides the placement and density of nodes on its own.

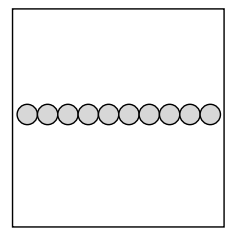

(a) FS10x1

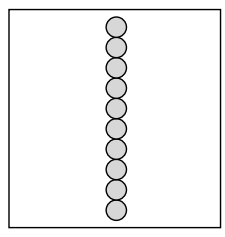

(b) FS1x10

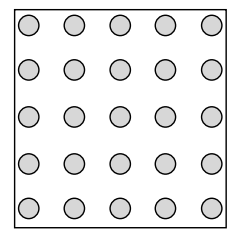

(c) $\mathrm{FS} 5 \times 5$

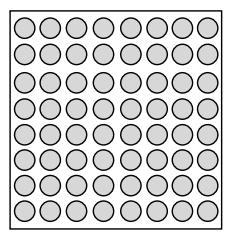

(d) $\mathrm{FS} 8 \times 8$

Figure 8. Hidden-node layouts for the original HyperNEAT. This figure shows (a) a horizontal configuration of hidden nodes, (b) a vertical arrangement, and two (c,d) grid configurations. 
Experimental parameters for this experiment and all other experiments in this article are given in Appendix 1.

\subsection{Dual Task Results}

All results are averaged over 20 runs. Figure 9 shows the training performance over generations for the HyperNEAT variants on the dual task domain. ES-HyperNEAT solved the domain in all runs and took on average 33 generations $(\sigma=31)$, whereas the best-performing fixed-substrate variant, FS5x5, found a solution in only 13 out of 20 runs. The difference in average final performance is significant $(p<0.001$ according to the Student's $t$-test $)$.

The second HyperNEAT thresholding method, HyperNEAT-LEO, was seeded with global locality [62], which should allow HyperNEAT to create more-sparsely connected networks. Indeed, adding the LEO increases the average maximum fitness for all fixed-substrate HyperNEAT setups significantly $(p<0.001)$ (graphs not shown). The fixed-substrate approaches with LEO $10 \times 1,1 \times$ $10,5 \times 5$, and $8 \times 8$ find a solution in 20,18,17, and 19 runs, respectively. The best fixed-substrate approach with LEO $(10 \times 1)$ took 28 generations on average $(\sigma=52)$, which is slightly, though not significantly, faster than ES-HyperNEAT $(p=0.68)$. However, the worst-performing fixedsubstrate approach with LEO $(5 \times 5)$ took 101 generations on average $(\sigma=116)$ when successful, which is significantly longer than ES-HyperNEAT $(p<0.05)$. This result highlights that even though HyperNEAT-LEO improves on the performance of regular HyperNEAT, the need to decide the placement of nodes (which is removed with ES-HyperNEAT) remains a potential liability.

These results also suggest that a multimodal domain benefits from the ability of both ES-HyperNEAT and HyperNEAT-LEO to generate more-sparsely connected ANNs than the original HyperNEAT with uniform thresholding, which an analysis of the evolved ANNs confirms. An example of three ANN solutions generated by ES-HyperNEAT and the CPPNs that encode them is shown in Figure 10. While ES-HyperNEAT is able to find a greater variation in connectivity for different neurons, the networks created by the original HyperNEAT are generally fully or near-fully connected.

Interestingly, the average CPPN complexity of solutions discovered by the best-performing setup for regular HyperNEAT $(5 \times 5)$ is at 9.7 hidden nodes $(\sigma=6.7)$, almost six times higher than CPPN solutions by ES-HyperNEAT, which have 1.65 hidden nodes on average $(\sigma=2.2)$. It is also three times higher than CPPN solutions for the best-performing HyperNEAT-LEO setup $(10 \times 1)$, which have 3.05 hidden nodes on average $(\sigma=2.25)$. These results indicate that regular HyperNEAT requires more effort to find solutions than either ES-HyperNEAT or HyperNEAT-LEO.

\section{Experiment 2: Maze Navigation}

To evolve controllers for more complicated tasks will require a neuroevolution method that benefits from previously discovered partial solutions to find the final solution. While direct encodings like

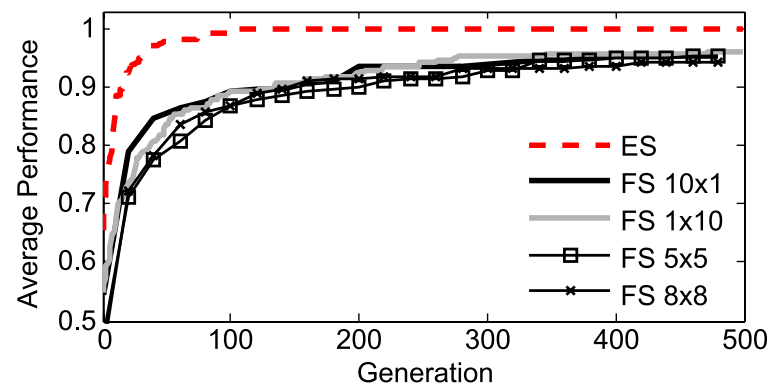

Figure 9. Average performance. The average best fitness over generations is shown for the dual task domain for the different HyperNEAT substrates, which are averaged over 20 runs. The main result is that ES-HyperNEAT significantly outperforms the original HyperNEAT in a multimodal domain. 

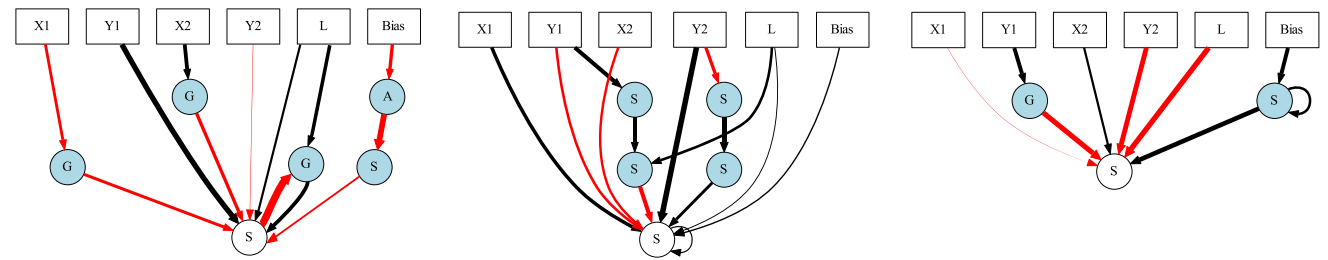

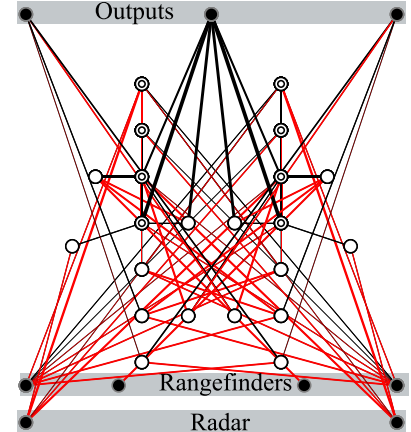

(a)
ANN: $22 \mathrm{n}, 84 \mathrm{c}$

CPPN: $5 \mathrm{n}, 13 \mathrm{c}$

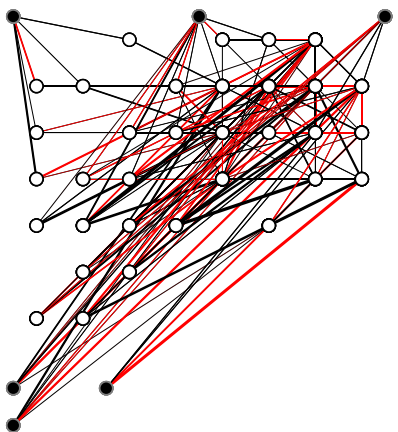

(b)

ANN: $33 \mathrm{n}, 143 \mathrm{c}$

CPPN: 4 n, $14 \mathrm{c}$

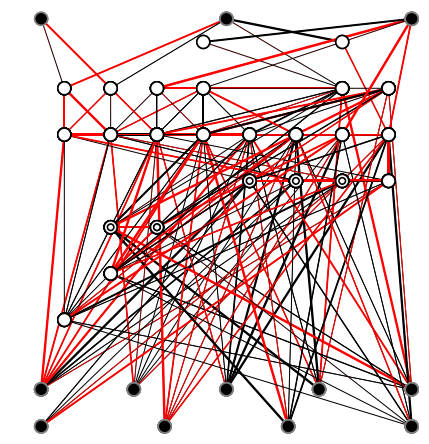

(c)
ANN: $24 \mathrm{n}, 156 \mathrm{c}$ CPPN: 2 n, 9 c

Figure 10. ES-HyperNEAT ANN solutions and their underlying CPPNs. Three ANN solutions (bottom) and the CPPNs (top) that encode them are shown together with the numbers of hidden neurons $(n)$ and connections (c). ES-HyperNEAT evolves a variety of different ANNs, showing varying degrees of symmetry and network connectivity. In the electronic version, positive connections are dark (black), whereas negative connections are light (red). Line width corresponds to connection strength. Hidden nodes with self-recurrent connections are denoted by a smaller concentric circle. CPPN activation functions are denoted by $G$ for Gaussian, $S$ for sigmoid, and $A$ for absolute value. The CPPNs receive the length $L$ of the queried connection as an additional input.

NEAT allow the network topology to complexify over generations, leading to increasingly sophisticated behavior, they suffer from the problem of reinvention. That is, even if different parts of the solution are similar, they must be encoded and therefore discovered separately.

HyperNEAT alleviated this problem by allowing the solution to be represented as a pattern of parameters, rather than requiring each parameter to be represented individually. However, because regular HyperNEAT tends to produce fully connected ANNs [7], it likely takes the entire set of ANN connection weights to represent a partial task solution, while ES-HyperNEAT should be able to elaborate on existing structure because it can increase the number of connections and nodes in the substrate during evolution, as suggested by Hypothesis 3.

To test this third hypothesis on when ES-HyperNEAT provides an advantage, a task is needed in which a solution is difficult to evolve without crossing several intermediate milestones. One such task is the deceptive maze navigation domain introduced by Lehman and Stanley [32]. In this domain (Figure 11), a robot controlled by an ANN must navigate in a maze from a starting point to an end point in a fixed time. The robot has five rangefinders that indicate the distance to the nearest wall within the maze, and four pie-slice radar sensors that fire when the goal is within the pie slice. The experimental setup follows the one described in Section 6.1 with the same substrate configuration and sensor layout (Figure 7). The agent thus sees walls with its rangefinders and the goal with its radars.

If fitness is rewarded proportionally to how close the robot is to the goal at the end, cul-de-sacs in the maze that lead close to the goal but do not reach it are deceptive local optima [34]. Therefore, to reduce the role of such deception in this article, the fitness function $f$ rewards the agent explicitly for discovering stepping stones toward the goal:

$f= \begin{cases}10 & \text { if the agent is able to reach the goal, } \\ n+(1-d) & \text { otherwise, }\end{cases}$ 


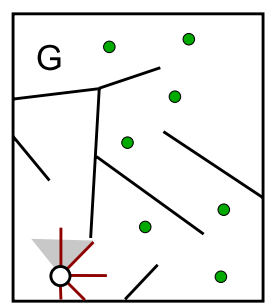

Figure II. Maze navigation. The goal of the agent in the maze navigation domain is to reach goal point $G$. Because the task is deceptive, the agent is rewarded for making incremental process toward the goal by following the waypoints.

where $n$ is the number of passed waypoints (which are not visible to the agent) and $d$ is the distance of the robot to the next waypoint scaled into the range $[0,1]$ at the end of the evaluation. The idea is that agents that can reach intermediate waypoints should make good stepping stones to those that reach further waypoints. ES-HyperNEAT should be able to elaborate more efficiently on agents that reach intermediate waypoints by gradually increasing their neural density.

\section{I Maze Navigation Results}

ES-HyperNEAT performed significantly better than the other variants in the maze navigation domain $(p<0.001)$ (Figure 12a) and finds a solution in 19 out of 20 runs in 238 generations on average when successful $(\sigma=262)$. The default setup for the original HyperNEAT, FS10x1, reaches a significantly higher average maximum fitness than the vertically arrangement FS1x10 $(p<0.001)$ or the gridlike setup FS8x8 $(p<0.05)$. The significantly lower performance of the vertical node arrangement (FS1x10) highlights the challenge of deciding the best positions for the hidden nodes and shows that certain substrate configurations make finding an effective pattern of weights more difficult, as suggested by Hypothesis 2 .

The differing performance of evolvable-and fixed-substrate HyperNEAT can also be appreciated in how frequently they solve the problem perfectly (Figure 12b). ES-HyperNEAT significantly outperforms all fixed-substrate variants and finds a solution in $95 \%$ of the 20 runs. FS10x1 solves the domain in $45 \%$ of runs, whereas the vertical arrangement of the same number of nodes (FS1x10) degrades performance significantly $(p<0.001)$, also not finding a solution in any of the runs. FS5x5 finds a solution in $20 \%$ of all runs. Interestingly, uniformly increasing the number of hidden nodes to 64 for FS8x8, which might be hypothesized to help, in fact degrades performance significantly $(p<0.001)$, not finding a solution in any of the runs.

Extending the original HyperNEAT with the LEO and global locality seeding [62] increases its average maximum fitness for all but the FS8x8 setup significantly $(p<0.001)$ (graphs not shown). The best-performing HyperNEAT-LEO setup (FS10x1) finds a solution in 17 out of 20 runs, in

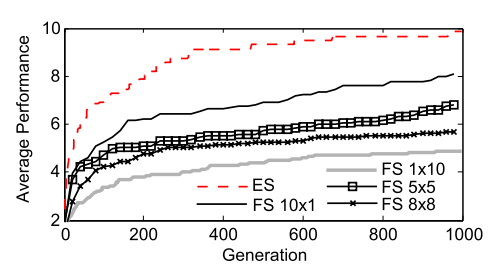

(a) Maze Navigation Training

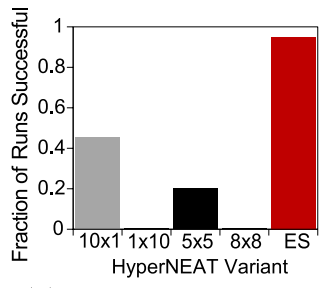

(b) Successful Maze Runs

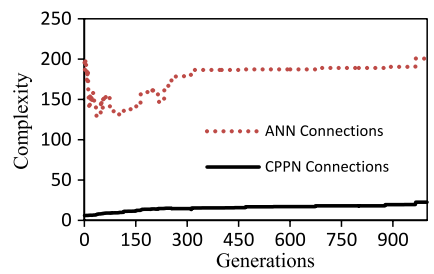

(c) Champion Complexity

Figure 12. Average performance and champion complexity. The average best fitness over generations is shown for the maze navigation domain (a) for the different HyperNEAT variants, which are averaged over 20 runs. The fraction of 20 runs that successfully solve the maze navigation domain is shown in (b) for each of the HyperNEAT variants after I,000 generations. The average number of connections of the champion ANNs produced by ES-HyperNEAT and the number of connections of the underlying CPPNs are shown in (c). Increasing CPPN complexity shows a positive (and significant) correlation with an increase in ANN substrate complexity. 
531 generations on average when successful $(\sigma=262)$. While LEO improves HyperNEAT's performance, the best-performing fixed-substrate approach even with LEO still performs significantly worse than ES-HyperNEAT $(p<0.05)$. This result suggests that the maze navigation domain depends not only on networks with limited connectivity (like the dual task domain in the previous section), but also on a method that benefits from building on previously discovered partial solutions to find the final solution.

In ES-HyperNEAT, there is a significant positive correlation $(r=0.95, p<0.001$ according to Spearman's rank correlation coefficient) between the number of connections in the CPPN and in the resulting ANN (Figure 12c). This trend indicates that the substrate evolution algorithm may tend to create increasingly complex indirectly encoded networks even though it is not explicitly designed to do so (e.g., like regular NEAT). The complexity of ANN (substrate) solutions (242 connections on average) is more than nine times greater than that of the underlying CPPNs (25 connections on average), which suggests that ES-HyperNEAT can encode large ANNs from compact CPPN representations.

The conclusion is that evolving the substrate can significantly increase performance in tasks that require incrementally building on stepping stones. The next two sections examine how this result comes about.

\subsection{Example Solution Lineage}

To gain a better understanding of how an indirect encoding like ES-HyperNEAT elaborates a solution over generations, additional evolutionary runs in the maze navigation domain were performed with sexual reproduction disabled (i.e., every $\mathrm{CPPN}$ has only one ancestor). This change facilitates analyzing the lineage of a single champion network. Disabling sexual reproduction did not result in a significant performance difference.

An example of four milestone ANNs in the lineage of a solution and the CPPNs that encode them is shown in Figure 13. All ANNs share common geometric features: Most prominent are the symmetric network topology and denser regions of hidden neurons resembling the shape of an " $\mathrm{H}$ " (except the second ANN). Between generations 24 and 237 the ANN evolves from not being able to reach the first waypoint to solving the task.

The solution discovered at generation 237 shows a clear holistic resemblance to generation 106 despite some general differences. Both networks have strong positive connections to the three output neurons that originate at slightly different hidden-node locations. This slight shift is due to a movement of information within the hypercube for which ES-HyperNEAT can nevertheless compensate, as suggested by Hypothesis 2 . The number of connections gradually increases from 184 in generation 24 to 356 in generation 237, indicating the incremental elaboration on existing ANN structure, as suggested by Hypothesis 3. Interestingly, the final ANN solves the task without feedback from its pie-slice sensors.

Figure 13 also shows that ES-HyperNEAT can encode large ANNs from compact CPPN representations. The solution ANN with 40 hidden neurons and 356 connections is encoded by a much smaller CPPN with only five hidden neurons and 18 connections.

In contrast to direct encodings like NEAT $[53,55]$, genotypic CPPN mutations can have a more global effect on the expressed ANN patterns. For example, changes in only four CPPN weights are responsible for the change in topology from the second to the third ANN milestone. Other solution lineages followed similar patterns, but single neuron or connection additions to the substrate do also sometimes occur.

\subsection{Initializing Regular HyperNEAT with a Solution ES-HyperNEAT Substrate}

One might speculate that ES-HyperNEAT outperforms the original HyperNEAT only because it finds a better substrate and not because it can compensate for movement of information within the hypercube or because it can incrementally build on existing ANN structure. To test this hypothesis, 20 additional runs were performed, in which HyperNEAT was given the solution substrate generated by ES-HyperNEAT in Figure 13d. 

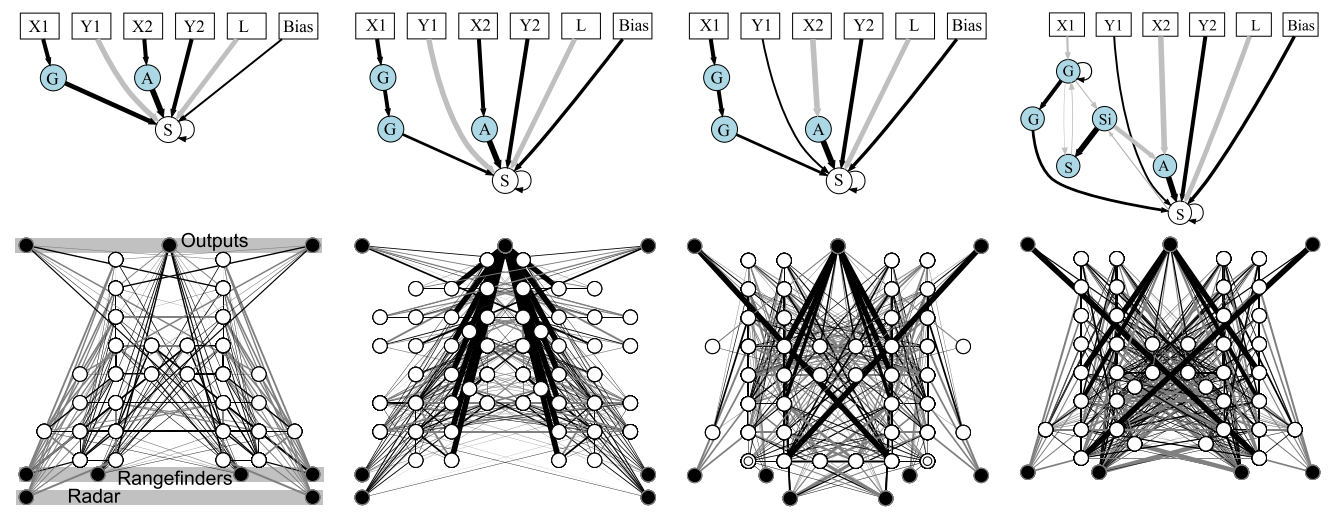

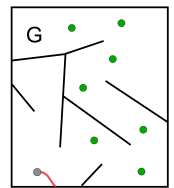

(a) Generation 24 ANN: 30 n, $184 \mathrm{c}$ CPPN: $2 \mathrm{n}, 9 \mathrm{c}$ fitness $=0.85$

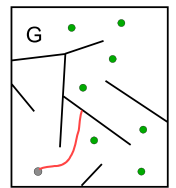

(b) Generation 30 ANN: $52 \mathrm{n}, 280 \mathrm{c}$ CPPN: $3 \mathrm{n}, 10 \mathrm{c}$ fitness $=0.93$

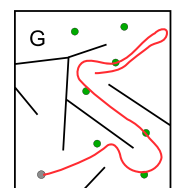

(c) Generation 106 ANN: $42 \mathrm{n}, 310 \mathrm{c}$ CPPN: $3 \mathrm{n}, 10 \mathrm{c}$ fitness $=5.96$

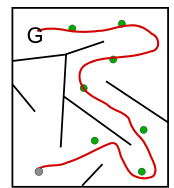

(d) Generation 237 ANN: $40 \mathrm{n}, 356 \mathrm{c}$ CPPN: $5 \mathrm{n}, 18 \mathrm{c}$ fitness $=10.00$

Figure 13. ANN milestones and underlying CPPNs together with the agent's behavior from a single maze solution lineage. Four ANN milestones (bottom) and the CPPNs (top) that encode them are shown together with the numbers of hidden neurons $(n)$ and connections (c). Fitness $f$ is also shown. Positive connections are dark, whereas negative connections are light. Line width corresponds to connection strength. Hidden nodes with self-recurrent connections are denoted by a smaller concentric circle. CPPN activation functions are denoted by $G$ for Gaussian, $S$ for sigmoid, Si for sine, and $A$ for absolute value. The CPPNs receive the length $L$ of the queried connection as an additional input. The gradual increase in substrate connections indicates an increase of information in the hypercube, which in turn leads to an increase in performance.

HyperNEAT solved the task in $40 \%$ of all runs and reached an average maximum fitness of 7.30 $(\sigma=2.33)$. It performed slightly worse than the FS10x1 setup, which solved the task in $45 \%$ of all runs, and significantly worse than ES-HyperNEAT $(p<0.001)$.

Thus the results confirm the hypothesis that ES-HyperNEAT's better performance is not only due to the topography of its generated substrates. The original HyperNEAT does not have the ability to modify the locations of the hidden nodes, which, as suggested by Hypothesis 2, makes finding an effective pattern of weights more difficult. Additionally, even with a substrate generated by ES-HyperNEAT, HyperNEAT is not able to elaborate further on the existing structure, because it takes the entire set of ANN connection weights to represent a partial solution.

\subsection{Evolvability Analysis}

Kirschner and Gerhart [31] define evolvability as “an organism's capacity to generate heritable phenotypic variation." The highly evolvable representations found in biological systems have allowed natural evolution to discover a great variety of diverse organisms. Thus facilitating a representation's effective search (i.e., its evolvability) is an important research direction in evolutionary computation [43].

The dual task (Section 6) and the maze navigation domain (Section 7) suggest that the original HyperNEAT fails to elaborate on existing ANN structure because it likely consumes the entire set of connection weights to represent an intermediate solution. Furthermore, small mutations in the CPPN can cause a shift in the location of information within the hypercube for which the original HyperNEAT cannot compensate, making such evolved individuals fragile. However, ES-HyperNEAT should be more robust, because it can compensate for shifts in the CPPN pattern by following the movement of information within the hypercube (Hypothesis 2). This ability and the fact that 
ES-HyperNEAT can elaborate on existing ANN structure (Hypothesis 3) should allow ES-HyperNEAT to more easily generate individuals whose offspring exhibit diverse functional behaviors, and thus more heritable phenotypic variation. To demonstrate this capability, the evolvability of the different representations needs to be measured.

Kirschner's definition reflects a growing consensus in biology that the ability to generate behavioral variation is fundamental to evolvability $[31,41,63]$. Therefore, following Lehman and Stanley [33], an individual's evolvability in this article is estimated by generating many children from it and then measuring the degree of behavioral variation among those offspring. In effect, this measure quantifies how well the underlying encoding enables behaviorally diverse mutations. To measure variation, a behavioral distance measure is needed. Following Lehman and Stanley [33], behavioral distance in the maze navigation domain is measured in this analysis by the Euclidean distance between the ending positions of two individuals. Evolvability is measured every 50 generations, when each individual in the population is forced to create 200 offspring by asexual reproduction. A greedy algorithm calculates the evolvability by adding each individual to a list of behaviors if its behavioral distance to all other individuals already in the list is higher than a given threshold. Thus the number of added behaviors is an indicator of the individual's ability to generate behavioral variation (i.e., its evolvability).

It is important to note that a random genetic mapping would likely not enable behaviorally diverse mutations and therefore would have a low evolvability. Most of the networks produced would be nonfunctional or display trivial behaviors (just going forward, spinning in circles, etc.), thus ending in similar parts of the maze. The results in the previous section (Figure 12a) showed that evolving a successful maze navigator is a challenging task, which supports the hypothesis that generating nontrivial behaviors through a random mapping is unlikely.

Figure 14 shows the average evolvability of the best-performing fixed-substrate variant FS10x1 compared with ES-HyperNEAT. The main result is that ES-HyperNEAT shows a significantly $(p<$ 0.001) higher evolvability across all generations, further explaining its better performance in the domains presented in this article.

\section{Experiment 3: Retina Problem}

Modularity likely plays an important role in the evolvability of complex biological organisms $[7,13$, 23, 29]. Lipson [35] defines functional modularity as the structural localization of function, which allows parts of a solution to be optimized independently [29]. Because modularity enhances evolvability and allows natural systems to scale to tasks of high complexity, it is an important ingredient in evolving complex networks, which is a major goal in the field of GDSs. ES-HyperNEAT should more easily evolve modular ANNs than the original fixed-substrate HyperNEAT, because it has the capability to start the evolutionary search with a bias toward locality and certain canonical ANN topographies, as suggested by Hypothesis 4 .

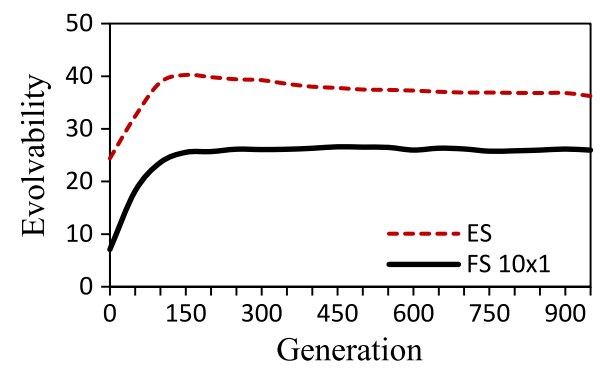

Figure 14. Comparing the evolvability of fixed-substrate and evolvable-substrate HyperNEAT. ES-HyperNEAT exhibits a significantly higher evolvability throughout all generations. The results are averaged over 20 runs. 


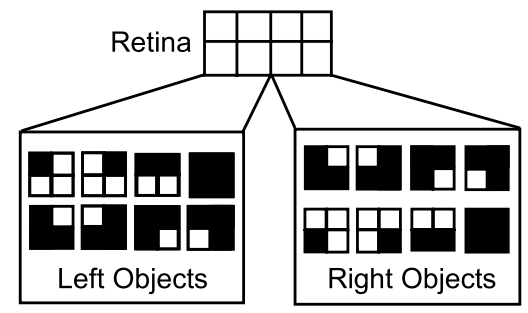

Figure 15. Retina problem. The artificial retina consists of $4 \times 2$ pixels that constitute the inputs to the ANN. Eight out of 16 possible patterns are considered left objects. The same is true for the right four pixels, though the eight valid patterns are different. The picture is adapted from Kashtan and Alon [29].

Following Verbancsics and Stanley [62] and Clune et al. [7], the modular domain in this section is a modified version of the retina problem, originally introduced by Kashtan and Alon [29]. The goal of the ANN is to identify a set of valid $2 \times 2$ patterns on the left and right sides of a $4 \times 2$ artificial retina (Figure 15). That is, the ANN must independently decide, for each pattern presented to the left and right sides of the retina, if that pattern is a valid left or right pattern, respectively. Thus it is a good test of the ability to evolve modular structures, because the left and right problem components are ideally separated into different functional structures. The ANN setup is given below in Section 8.3.

While the original HyperNEAT approach and also the direct NEAT encoding perform poorly in generating modular ANNs [7], HyperNEAT-LEO [62] showed that allowing HyperNEAT to evolve the pattern of weights independently from the pattern of connection expression, while seeding HyperNEAT with a bias toward local connectivity, allows modular structures to arise naturally. Furthermore, the LEO achieved the best results in the retina left \& right task by only seeding with the concept of locality along the $x$ axis [62]. That is, the seed CPPN starts with a Gaussian node $G$ that receives $x_{1}-x_{2}$ as input and is connected to the LEO output. Therefore $G$ peaks when the two coordinates are the same, thereby seeding the CPPN with a concept of locality. This result makes sense because the retina problem is distributed along the horizontal axis (Figure 15).

\section{I Extending ES-HyperNEAT with the LEO}

Because ES-HyperNEAT does not require any special changes to the traditional HyperNEAT CPPN, enhancements like the LEO can in principle also be incorporated into ES-HyperNEAT. Thus extending ES-HyperNEAT with an LEO is straightforward and should combine the advantages of both methods: Evolving modular ANNs should be possible wherein the placement and density of hidden nodes are determined solely from implicit information in the hypercube. In this combined approach, once all connections are discovered by the weight-choosing approach (Section 5), only those are kept whose LEO output is greater than zero. In fact, the idea that geometric concepts such as locality can be imparted to the CPPN opens an intriguing opportunity to go further than the LEO locality seed. That is, it is also possible to start the evolutionary search in ES-HyperNEAT with a bias toward certain ANN topographies (which is not possible with HyperNEAT or HyperNEAT-LEO), as explained in Section 8.2.

\subsection{ES-HyperNEAT Geometry Seeding}

Because the pattern output by the CPPN in ES-HyperNEAT is a kind of language for specifying the locations of expressed connections and nodes, ES-HyperNEAT can be seeded to begin with a bias toward certain ANN structures (e.g., ANNs with multiple hidden layers and connected inputs and outputs) that should facilitate the evolutionary search. Especially in the initial generations, ES-HyperNEAT runs the risk of being trapped in local optima where high fitness can be achieved only by incorporating a subset of the available inputs. The new idea introduced in this article is to start the evolutionary search with a bias toward certain ANN topographies, which provide a mechanism for emulating key biases in the natural world that are provided ultimately by physics. For example, 
evolution could be seeded with an ANN topography that resembles the organization of the cortical columns found in the human brain [51], potentially allowing higher cognitive tasks to be solved.

Providing such bias means escaping the black box of evolutionary optimization to provide a kind of general domain knowledge. Even though ES-HyperNEAT could in principle discover the appropriate ANN topography by itself, biasing the search with a good initial topography should thus make the search less susceptible to local optima. While ES-HyperNEAT can modify and elaborate on such initial ANN structure, the original HyperNEAT and HyperNEAT-LEO would likely not benefit from geometry seeding, because they cannot compensate for movement of information within the hypercube, and certain structures are a priori not representable if the nodes are not placed in the correct locations.

Figure 16 shows a CPPN that combines seeded locality and seeded geometry. In addition to the Gaussian node $G_{1}$ that specifies locality along the $x$ axis [62], a second Gaussian node $G_{2}$ is added that receives $y_{1}-y_{2}+b$, where $b$ is a bias, as input (Figure 16) and therefore creates horizontal stripes of differing weights running through the hypercube along $y_{1}$. Interestingly, changing the bias input of $G_{2}$ thus can immediately create $A N N s$ with more or fewer bidden layers. In the experiments reported here a bias weight of 0.33 was chosen, resulting in initial ANNs with two hidden layers, which is similar to the setup of the fixed-substrate variant explained in the next section. It is important to note that most connections will initially not be expressed, because of the locality seeding. However, slight perturbations of the seed in the initial generation provide a variety of local connectivity patterns and ANN topographies. That the organization of a locally connected two-hidden-layer substrate can be entirely described by two new hidden nodes in the initial CPPN suggests the power of the encoding and the expressiveness that is possible when seeding ES-HyperNEAT.

\subsection{Retina Problem Setup}

The substrate, which is based on setups of Verbancsics and Stanley [62] and Clune et al. [7], has eight input nodes and two output nodes (Figure 17). Fixed-substrate HyperNEAT has two layers of hidden nodes, with four hidden nodes each. Note that the substrate has three dimensions $(x, y, z)$. The ANN substrate inputs receive either -3.0 or 3.0, depending on the state (e.g., off or on) for each retina input. The left and right outputs specify the classification for the left and right retinas, respectively, where values close to 1.0 indicate valid patterns. Values close to -1.0 indicate an invalid pattern. Six different HyperNEAT approaches are compared to isolate the effects of the LEO and the hidden-layer seeding:

- In the ES-HyperNEAT approach the placement and density of the hidden nodes and their connections to the input and output nodes are determined entirely from the CPPN by the algorithm in Section 3 without any seeding.

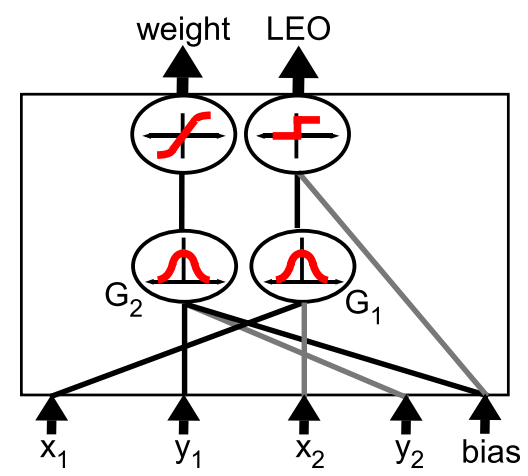

Figure 16. X-locality and geometry seeding. The CPPN is initialized with two Gaussian hidden nodes $G_{1}$ and $G_{2}$ that take as input $x_{1}-x_{2}$ and $y_{1}-y_{2}+b$, respectively. Whereas $G_{1}$ is connected to the LEO with a bias of $-I, G_{2}$ connects to the weight output. $G_{1}$ peaks when $x_{1}$ and $x_{2}$ are the same, thereby seeding the initial CPPNs with locality along the $x$ axis. $G_{2}$ creates horizontal stripes of differing weights running through the hypercube, which induces the expression of multiple hidden layers in the decoded ANN. Positive connections are dark, and negative connections are light. 


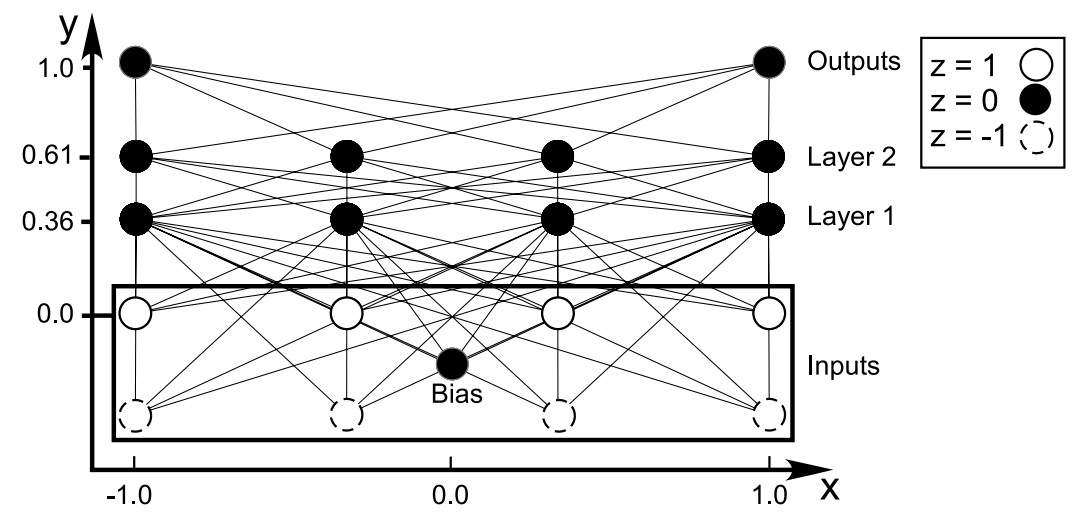

Figure 17. Substrate configuration. The substrate consists of four layers with the inputs at $y=0.0$, the first hidden layer at $y=0.37$, a second hidden layer at $y=0.6 \mathrm{I}$, and the outputs at $y=1.0$. Note that this substrate has three dimensions. The $z$ coordinates are indicated by the different circle patterns. This substrate configuration is derived from the setups of Verbancsics and Stanley [62] and Clune et al. [7], which established such a three-dimensional setup as standard for the retina problem.

- ES-HyperNEAT-LEO extends ES-HyperNEAT with LEO, which should facilitate the evolution of modular ANNs.

- ES-HyperNEAT with geometry seeding tests ES-HyperNEAT's ability to take advantage of initial geometric seeding through the CPPN. The seed, shown in Figure 16, creates ANNs with two hidden layers with four neurons each, corresponding to the fixed-substrate retina setup (Figure 17). Note that the functionality of the LEO is disabled in this setup.

- ES-HyperNEAT-LEO with geometry seeding tests the hypothesis that both extensions, LEO with locality seeding and geometric seeding (Figure 16), should be complementary in increasing ES-HyperNEAT's ability to generate modular networks for complicated classification problems.

- Following Verbancsics and Stanley [62], FS-HyperNEAT-LEO with x-locality seeding is the original HyperNEAT approach with a fixed substrate and an additional LEO.

- In the FS-HyperNEAT-LEO with geometry seeding approach the original Hyper-NEAT is also seeded with $x$-locality and initial weight patterns (Figure 16) that intersect the positions of the fixed hidden nodes (Figure 17). The hypothesis is that the additional geometric seeding should not significantly increase fixed-substrate HyperNEAT's performance, because small variations in the initial seed will disrupt the alignment between the CPPN-expressed pattern and hidden-node positions, for which the original HyperNEAT cannot compensate.

Note that the seed CPPN for all approaches lacks direct connections from the inputs to the weight output in the CPPN. However, mutations on the seed to create the initial generation can connect arbitrary inputs to arbitrary outputs. This setup has shown generally better performance than starting with a fully connected CPPN. The fitness, which is computed the same way for all approaches, is inversely proportional to the summed distance of the outputs from the correct values for all 256 possible patterns: $F=1000.0 /\left(1.0+E^{2}\right)$, where $E$ is the error. Other parameters are detailed in Appendix 1.

\subsection{Results}

All results are averaged over 40 runs. Figure 18 shows the training performance over generations for the different HyperNEAT variants and how frequently they solve the problem perfectly (i.e., correctly classify $100 \%$ of the patterns). Because FS-HyperNEAT-LEO succeds in almost every 
run after 5,000 generations [62], to highlight the differences in the HyperNEAT variants, runs were performed for a much shorter period of 2,000 generations (Figure 18b).

While ES-HyperNEAT solves the retina domain in only $30 \%$ of the runs, augmenting ES-HyperNEAT with LEO and geometry seeding improves the chance of finding a solution to $57 \%$. ES-HyperNEAT-LEO with geometry seeding reaches a significantly higher average best fitness and finds a solution significantly faster than the other variants $(p<0.05)$, confirming Hypothesis 4 and the advantages of both ES-HyperNEAT extensions for the evolution of modular ANNs. The performance of the original FS-HyperNEAT with LEO, on the other hand, decreases from finding a solution in 30\% of the runs to only finding the solution in $25 \%$ when seeded with geometry, confirming that only ES-HyperNEAT can take advantage of such geometry seeding.

Surprisingly, just extending ES-HyperNEAT with an LEO alone does not increase performance but instead decreases it slightly (though not significantly). Especially in the first 1,000 generations, there is almost no increase in performance (Figure 18a), which suggests that pruning connections based on the amount of information in the hypercube and additionally through the LEO without any geometry seeding hinders the evolution of functional networks (i.e., ANNs with paths from the input to the output neurons). Additionally, only seeding with geometry but not extending ES-HyperNEAT with the LEO (i.e., ES-HyperNEAT with only geometry seeding) also decreases performance, which is likely due to the increased crosstalk in the more fully connected ANNs.

A closer look at the structure of some final solutions gives insight into how ES-HyperNEATLEO can elaborate on initial geometric seeding (Figure 19). ES-HyperNEAT-LEO can successfully build on the initial structure, creating networks of varying complexity and resemblance to the initial seed (Figure 16). Modularity is the prevailing pattern (Figure 19a,b,e), but non-modular ANNs also emerge (Figure 19c). While most networks display a high degree of symmetry (Figure 19a-c), reflecting the symmetry in the retina patterns (Figure 15), less symmetric ANNs are also discovered (Figure 19d,e).

The main result is that it is the combination of LEO and geometry seeding that allows ES-HyperNEAT more easily to evolve ANNs for the modular retina problem. The original HyperNEAT approach, on the other hand, cannot take full advantage of those extensions and performs significantly worse, even though it too benefits from the LEO.

\section{Discussion and Future Work}

The central insight in this article is that a representation that encodes the pattern of connectivity across a network (such as in HyperNEAT) automatically contains implicit clues on where the nodes should be placed to best capture the information stored in the connectivity pattern. Experimental results show that ES-HyperNEAT significantly outperforms the original HyperNEAT while taking a

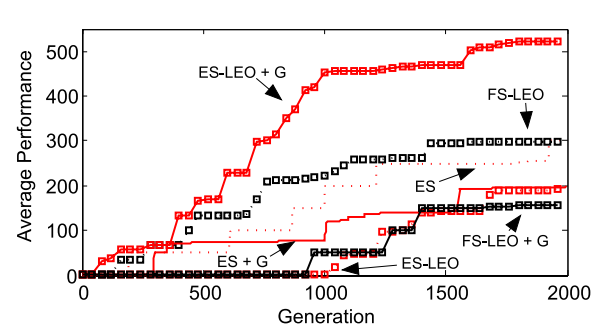

(a) Retina Training

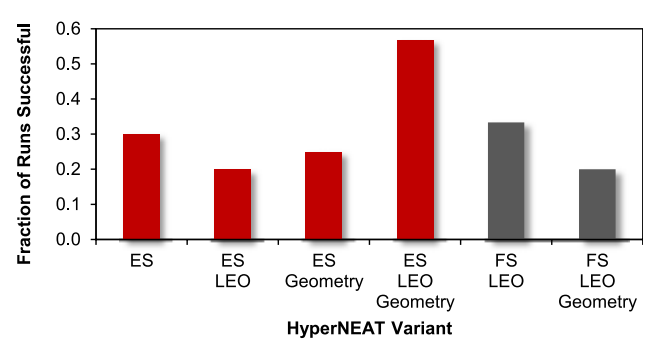

(b) Successful Runs

Figure 18. Average performance. The average best fitness over generations is shown for the retina domain (a) for the different HyperNEAT variants, which are averaged over 40 runs. The fraction of 40 runs that successfully solve the domain is shown in (b) for each of the HyperNEAT variants after 2,000 generations. The main result is that ES-HyperNEAT-LEO with geometry seeding significantly outperforms all other approaches. 


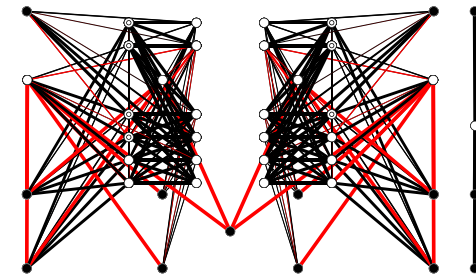

(a) 55 neurons, 198 connections

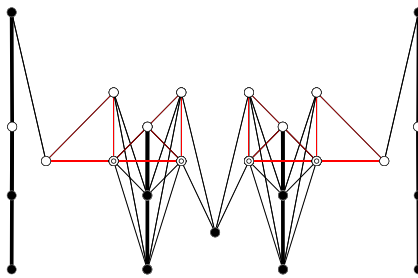

(b) 25 neurons, 54 connections

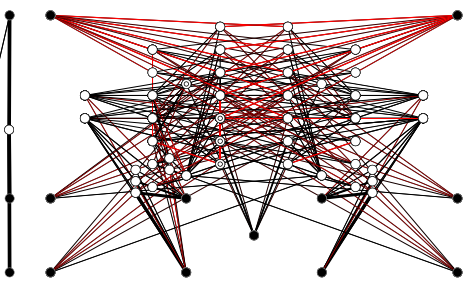

(c) 69 neurons, 201 connections

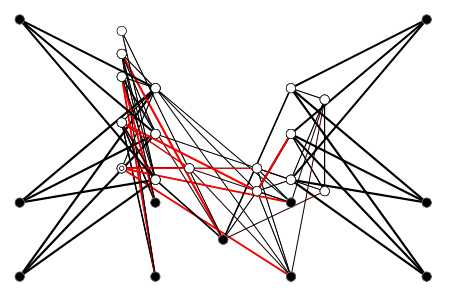

(d) 27 neurons, 72 connections

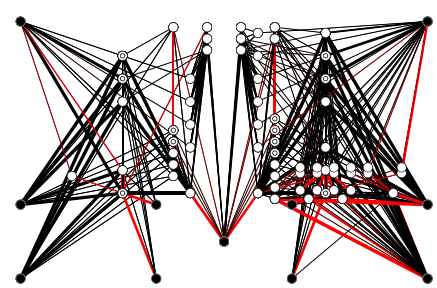

(e) 77 neurons, 368 connections

Figure 19. Example connectivity patterns from ES-HyperNEAT-LEO with geometry seeding in a modular domain. Modularity is commonly found when ES-HyperNEAT-LEO is seeded with the geometry seed $(a, b)$. Once modularity is found, the regularities needed to solve the task for each module can be discovered in the weight pattern. ES-HyperNEAT-LEO evolves a variety of different ANNs with more or fewer hidden neurons and connections. Non-modular ANNs that solve the task are also discovered (c), although with less frequency. Hidden nodes with recurrent connections are denoted by a smaller concentric circle. In the electronic version, positive connections are dark (black), and negative connections are light (red).

step toward more biologically plausible ANNs and significantly expanding the scope of neural structures that evolution can discover. This section explores the implications of this capability and its underlying methodology.

\section{I Dictating Node Locations}

The convention in HyperNEAT of the last several years that the user would simply decide a priori where the nodes belong evaded the deeper mystery about how connectivity relates to node placement. As suggested by Hypothesis 2, dictating the location of nodes makes it harder for the original HyperNEAT to represent the correct pattern, which the reported results in a variety of domains confirm. While ES-HyperNEAT can compensate for movement of information within the hypercube by expressing the hidden nodes at slightly different locations (e.g., Figure 13c,d), representing the correct pattern for the original HyperNEAT is more difficult. The significantly reduced performance of the vertical node arrangement FS1x10 in the maze navigation domain (Figure 12a) indicates that the more complex the domain, the more restrictive it is to have nodes at fixed locations.

One way to interpret the preceding argument is that the locations of useful information in the hypercube are where the nodes need to be. That way, the size of the brain is roughly correlated with its complexity. There is no need for a billion neurons to express a simple Braitenberg vehicle. Even if a billion neurons were summoned for the task, many of their functions would end up redundant, which geometrically means that large cross sections of the hypercube would be uniform, containing no useful information. The ES-HyperNEAT approach in this article is a heuristic attempt to formalize this notion and thereby correlate size with complexity. In this context, nodes become a kind of harbinger of complexity, proliferating where it is present and receding where it is not. Thus the solution to the mystery of the relationship between nodes and connections is that nodes are sentinels of complex connectivity; they are beacons of information in an infinite cloud of possible connections.

\subsection{Incrementally Building on Stepping Stones}

Previous work showed that ES-HyperNEAT and the original HyperNEAT exhibit similar performance in a simple navigation domain [44]. However, in the more complicated navigation domain presented 
here (Section 7), the best fixed-substrate HyperNEAT method (FS10x1) solves the domain in only $45 \%$ of runs. How can this poor performance be explained?

The problem is that the increased complexity of the domain requires incrementally building on previously discovered stepping stones. While direct encodings like NEAT $[56,58]$ can complexify ANNs over generations by adding new nodes and connections through mutation, the indirect HyperNEAT encoding tends to start with already fully connected ANNs [8], which take the entire set of ANN connection weights to represent a partial task solution. On the other hand, ES-HyperNEAT is able to elaborate on existing structure in the substrate during evolution (Figure 13), confirming Hypothesis 3. This result is important because the more complicated the task, the more likely that it will require a neuroevolution method that benefits from previously discovered stepping stones.

These results also explain why uniformly increasing the number of hidden nodes in the substrate does not necessarily increase HyperNEAT's performance. In fact, FS8x8 performs significantly worse than FS5x5, which is likely due to the increased crosstalk that each neuron experiences.

\subsection{Network Complexity}

An important question is whether the number of neurons created in ES-HyperNEAT networks is too large. In fact, in some cases the approach may find solutions with several times more nodes and connections than needed for the minimum solution. While the real test of this question will emerge from further research, perhaps the singular focus within the field of NE on absolute minimal structure is misplaced. When the products of evolution contain potentially billions of neurons as in nature, it is almost certainly necessary that an encoding that can reach such levels has the ability to solve particular problems with significant flexibility in the number of neurons in the solution. Of course, if a particular level of intelligence can be achieved with only a million neurons, then a solution with a billion would be undesirable. However, too much restriction on variation in the number of neurons is likely to be equally disruptive. In this situation, quibbling about a few dozen more or fewer neurons may be missing the forest for the trees. In a larger context, all of the networks reported in this work are small in a biological sense, as they should be.

It is important to keep in mind that the larger ES-HyperNEAT solutions are nevertheless optimized more quickly than their fixed-substrate counterparts. An indirectly encoded neuroevolution algorithm is not the same as a direct encoding like NEAT that complexifies by adding one node at a time to find just the right number of nodes for a task. The promise of the indirect encoding is rather to evolve very lange networks that would be prohibitive to such direct encodings, with thousands or more nodes. Figure $12 \mathrm{c}$ shows that the substrate evolution algorithm can actually create increasingly complex indirectly encoded networks even though it is not explicitly designed to do so (e.g., like regular NEAT) and that it can encode large ANNs from compact CPPN representations. One reason for this capability is often to increase the impact of adding new nodes and connections to the CPPN increases the complexity of the pattern it encodes. Because ES-HyperNEAT in effect attempts to match the structures it discovers in the hypercube to the complexity of the pattern within it, it makes sense that as the size of the CPPN increases, the complexity of the ANN substrate it encodes would generally increase as well.

ES-HyperNEAT's new capabilities and its higher evolvability (Figure 14) should enable more complex tasks to be solved in the future that require placing a large and unknown number of nodes, and the traversal of many stepping stones. The more complex the task, the more important it will be to free the user from the burden of configuring the substrate by hand. Also importantly, the approach has the potential to create networks from several dozen nodes up to several million or more, which will be necessary in the future to create truly intelligent systems.

\subsection{Geometry Seeding}

Whereas the original HyperNEAT only allowed seeding with a bias toward local connectivity [62], ES-HyperNEAT can also be seeded with a bias toward certain ANN topographies. The results in 
the retina problem confirm Hypothesis 4: The combination of LEO and geometry seeding allows the approach more easily to evolve modular ANNs, yet the original HyperNEAT approach cannot take full advantage of such a seed.

While the work of Verbancsics and Stanley [62] took a step in the direction of incorporating important geometric principles (e.g., locality) that are helpful to create structures that resemble those of nature, the geometry seeding presented here takes a step further. The idea of seeding with a bias toward certain types of structures is important because it provides a mechanism for emulating key biases in the natural world (such as the efficiency of modular separation) that are provided ultimately by physics.

As noted by Verbancsics and Stanley [62], the better performance with an initial bias toward locality suggests that the path to encoding locality is inherently deceptive with respect to the fitness function in the retina task. Such deception may turn out to be common when geometric principles such as locality that are conceptually orthogonal to the main objective are nevertheless essential to achieving the goal. Thus seeding with the right geometric bias may prove an important tool to avert deception in many domains.

Overall, ES-HyperNEAT thus advances the state of the art in neuroevolution beyond the original HyperNEAT and has the potential to create large-scale ANNs for more complicated tasks, such as robots driven by raw high-resolution vision, strategic game players, or robot assistants. For the field of AI, the idea that we are beginning to be able to reproduce some of the phenomena produced through natural evolution (e.g., compactly encoded regular and modular networks) at a high level of abstraction is important because the evolution of brains ultimately produced the seat of intelligence in nature.

Another interesting future research direction is to augment ES-HyperNEAT with the ability to indirectly encode plastic ANNs as a pattern of local learning rules [45]. Plastic networks can adapt and learn from past experience and, together with ES-HyperNEAT's ability to create complex ANNs, could allow the evolution of agents for more lifelike cognitive tasks.

\section{Conclusions}

This article has presented a novel approach to automatically deducing node geometry based on implicit information in an infinite-resolution pattern of weights. Evolvable-substrate HyperNEAT not only evolves the location of every neuron in the brain, but also can represent regions of varying density, which means resolution can increase holistically over evolution. To demonstrate this approach, ES-HyperNEAT evolved controllers for dual task, maze navigation, and modular retina problems. Analysis of the results and the evolved ANNs showed that the improved performance stems from ES-HyperNEAT's ability to evolve ANNs with partial and targeted connectivity, elaborate an existing ANN structure, and compensate for movement of information within the underlying hypercube. Additionally, ES-HyperNEAT can more easily evolve modular ANNs when biased toward locality and certain canonical ANN topographies. The main conclusion is thus that ES-HyperNEAT is a promising new approach that can create complex, regular, and modular ANNs as a function of neural geometry.

\section{Acknowledgments}

The authors would like to thank the Editor and anonymous reviewers for their valuable comments and suggestions, which were helpful in improving the article. This material is based upon work supported by the US Army Research Office under Award No. W911NF-11-1-0489 and the DARPA Computer Science Study Group (CSSG Phase 3) Grant No. N11AP20003. It does not necessarily reflect the position or policy of the government, and no official endorsement should be inferred.

\section{References}

1. Aaltonen, T., et al. (2010). Measurement of the top quark mass with dilepton events selected using neuroevolution at CDF. Physical Review D, 81, 030102. 
2. Angeline, P. J. (1995). Morphogenic evolutionary computations: Introduction, issues and examples. In J. R. McDonnell, R. G. Reynolds, \& D. B. Fogel (Eds.), Evolutionary Programming IV: The Fourth Annual Conference on Evolutionary Programming (pp. 387-401).

3. Angeline, P. J., Saunders, G. M., \& Pollack, J. B. (1994). An evolutionary algorithm that constructs recurrent neural networks. IEEE Transactions on Neural Networks, 5(1), 54-65.

4. Astor, J. S., \& Adami, C. (2000). A developmental model for the evolution of artificial neural networks. Artificial Life, 6(3), 189-218.

5. Bentley, P. J., \& Kumar, S. (1999). The ways to grow designs: A comparison of embryogenies for an evolutionary design problem. In Proceedings of the Genetic and Evolutionary Computation Conference (GECCO-1999) (pp. 35-43).

6. Bongard, J. (2002). Evolving modular genetic regulatory networks. In Proceedings of the 2002 Congress on Evolutionary Computation (CEC-2002), Vol. 2 (pp. 1872-1877).

7. Clune, J., Beckmann, B. E., McKinley, P., \& Ofria, C. (2010). Investigating whether HyperNEAT produces modular neural networks. In GECCO'10: Proceedings of the Genetic and Evolutionary Computation Conference (pp. 635-642).

8. Clune, J., Beckmann, B. E., Ofria, C., \& Pennock, R. T. (2009). Evolving coordinated quadruped gaits with the HyperNEAT generative encoding. In Proceedings of the IEEE Congress on Evolutionary Computation (CEC-2009) Special Section on Evolutionary Robotics.

9. Clune, J., Stanley, K., Pennock, R., \& Ofria, C. (2011). On the performance of indirect encoding across the continuum of regularity. IEEE Transactions on Evolutionary Computation, 15(3), 346-367.

10. Cybenko, G. (1989). Approximation by superpositions of a sigmoidal function. Mathematics of Control, Signals, and Systems, 2(4), 303-314.

11. D'Ambrosio, D., \& Stanley, K. O. (2007). A novel generative encoding for exploiting neural network sensor and output geometry. In Proceedings of the Genetic and Evolutionary Computation Conference (GECCO 2007).

12. Drchal, J., Koutnık, J., \& Šnorek, M. (2009). HyperNEAT controlled robots learn to drive on roads in simulated environment. In Proceedings of the IEEE Congress on Evolutionary Computation (CEC 2009).

13. Dürr, P., Mattiussi, C., \& Floreano, D. (2010). Genetic representation and evolvability of modular neural controllers. IEEE Computational Intelligence Magazine, 5(3), 10-19.

14. Eggenberger, P. (1997). Creation of neural networks based on developmental and evolutionary principles. In W. Gerstner, A. Germond, M. Hasler, \& J.-D. Nicoud (Eds.), Seventh International Conference on Artificial Neural Networks (ICANN-97) (pp. 337-342).

15. Eggenberger, P. (1997). Evolving morphologies of simulated 3D organisms based on differential gene expression. In Fourth European Conference on Artificial Life (pp. 205-213).

16. Finkel, R., \& Bentley, J. (1974). Quad trees: A data structure for retrieval on composite keys. Acta Informatica, 4(1), 1-9.

17. Floreano, D., Dürr, P., \& Mattiussi, C. (2008). Neuroevolution: From architectures to learning. Evolutionary Intelligence, 1(1), 47-62.

18. Gauci, J., \& Stanley, K. O. (2008). A case study on the critical role of geometric regularity in machine learning. In Proceedings of the Twenty-Third AAAI Conference on Artificial Intelligence (AAAI-2008).

19. Gauci, J., \& Stanley, K. O. (2010). Autonomous evolution of topographic regularities in artificial neural networks. Neural Computation, 22, 1860-1898.

20. Gomez, F. J., \& Miikkulainen, R. (1999). Solving non-Markovian control tasks with neuroevolution. In Proceedings of the 16th International Joint Conference on Artificial Intelligence (pp. 1356-1361).

21. Green, C. (2003-2006). SharpNEAT homepage. http://sharpneat.sourceforge.net/.

22. Gruau, F., Whitley, D., \& Pyeatt, L. (1996). A comparison between cellular encoding and direct encoding for genetic neural networks. In Genetic Programming 1996: Proceedings of the First Annual Conference (pp. 81-89).

23. Hansen, T. F. (2003). Is modularity necessary for evolvability? Remarks on the relationship between pleiotropy and evolvability. Biosystems, 69(2-3), 83-94. 
24. Harding, S., Miller, J. F., \& Banzhaf, W. (2010). Developments in Cartesian genetic programming: Self-modifying CGP. Genetic Programming and Evolvable Machines, 11(3-4), 397-439.

25. Harvey, I. (1993). The artificial evolution of adaptive behavior. Ph.D. thesis, School of Cognitive and Computing Sciences, University of Sussex, UK.

26. Hornby, G. S., \& Pollack, J. B. (2002). Creating high-level components with a generative representation for body-brain evolution. Artificial Life, 8(3), 223-246.

27. Jakobi, N. (1995). Harnessing morphogenesis. In Proceedings of Information Processing in Cells and Tissues (pp. 29-41).

28. Kandel, E. R., Schwartz, J. H., \& Jessell, T. M. (Eds.). (1991). Principles of neural science (3rd ed.). Amsterdam: Elsevier.

29. Kashtan, N., \& Alon, U. (2005). Spontaneous evolution of modularity and network motifs. Proceedings of the National Academy of Sciences of the United States of America, 102(39), 13773.

30. Khan, G. M., Miller, J. F., \& Halliday, D. M. (2011). Evolution of Cartesian genetic programs for development of learning neural architecture. Evolutionary Computation, 19(3), 469-523.

31. Kirschner, M., \& Gerhart, J. (1998). Evolvability. Proceedings of the National Academy of Sciences of the United States of America, 95(15), 8420.

32. Lehman, J., \& Stanley, K. O. (2008). Exploiting open-endedness to solve problems through the search for novelty. In S. Bullock, J. Noble, R. Watson, \& M. Bedau (Eds.), Proceedings of the Eleventh International Conference on Artificial Life (Alife XI).

33. Lehman, J., \& Stanley, K. O. (2011). Improving evolvability through novelty search and self-adaptation. In Proceedings of the 2011 IEEE Congress on Evolutionary Computation (pp. 2693-2700).

34. Lehman, J., \& Stanley, K. O. (2011). Abandoning objectives: Evolution through the search for novelty alone. Evolutionary Computation, 19(2), 189-223.

35. Lipson, H. (2007). Principles of modularity, regularity, and hierarchy for scalable systems. Journal of Biological Physics and Chemistry, 7(4), 125.

36. Martin, A. P. (1999). Increasing genomic complexity by gene duplication and the origin of vertebrates. The American Naturalist, 154(2), 111-128.

37. Miller, J. F. (2004). Evolving a self-repairing, self-regulating, French flag organism. In Proceedings of the Genetic and Evolutionary Computation Conference (GECCO-2004).

38. Miller, J. F., \& Thomson, P. (2000). Cartesian genetic programming. In Proceedings of the Third European Conference on Genetic Programming (pp. 121-132).

39. Mjolsness, E., Sharp, D. H., \& Reinitz, J. (1991). A connectionist model of development. Journal of Theoretical Biology, 152, 429-453.

40. Nolfi, S., \& Parisi, D. (1992). Growing neural networks. In C. G. Langton (Ed.), Proceedings of the Workshop on Artificial Life (ALIFE' '92).

41. Pigliucci, M. (2008). Is evolvability evolvable? Nature Reviews Genetics, 9(1), 75-82.

42. Reil, T., \& Husbands, P. (2002). Evolution of central pattern generators for bipedal walking in a real-time physics environment. IEEE Transactions on Evolutionary Computation, 6(2), 159-168.

43. Reisinger, J., Stanley, K. O., \& Miikkulainen, R. (2005). Towards an empirical measure of evolvability. In Genetic and Evolutionary Computation Conference (GECCO2005) workshop program (pp. 257-264).

44. Risi, S., Lehman, J., \& Stanley, K. O. (2010). Evolving the placement and density of neurons in the HyperNEAT substrate. In Proceedings of the Genetic and Evolutionary Computation Conference (GECCO 2010) (pp. 563-570).

45. Risi, S., \& Stanley, K. O. (2010). Indirectly encoding neural plasticity as a pattern of local rules. In S. Doncieux, B. Girard, A. Guillot, J. Hallam, J.-A. Meyer, \& J.-B. Mouret (Eds.), From Animals to Animats 11, Proceedings of the 11th International Conference on Simulation of Adaptive Behavior (pp. 533-543). Berlin: Springer.

46. Risi, S., \& Stanley, K. O. (2011). Enhancing ES-HyperNEAT to evolve more complex regular neural networks. In Proceedings of the Genetic and Evolutionary Computation Conference (GECCO 2011) (pp. 1539-1546). 
47. Rosenfeld, A. (1980). Quadtrees and pyramids for pattern recognition and image processing. In Proceedings of the 5th International Conference on Pattern Recognition (pp. 802-809).

48. Saravanan, N., \& Fogel, D. B. (1995). Evolving neural control systems. IEEE Expert: Intelligent Systems and Their Applications, 10(3), 23-27.

49. Secretan, J., Beato, N., D’Ambrosio, D. B., Rodriguez, A., Campbell, A., Folsom-Kovarik, J. T., \& Stanley, K. O. (2011). Picbreeder: A case study in collaborative evolutionary exploration of design space. Evolutionary Computation, 19(3), 373-403.

50. Secretan, J., Beato, N., D’Ambrosio, D. B., Rodriguez, A., Campbell, A., \& Stanley, K. O. (2008). Picbreeder: Evolving pictures collaboratively online. In CHI '08: Proceedings of the Twenty-sixth Annual SIGCHI Conference on Human Factors in Computing Systems (pp. 1759-1768).

51. Sporns, O. (2002). Network analysis, complexity, and brain function. Complexity, 8(1), 56-60.

52. Stanley, K. O. (2007). Compositional pattern producing networks: A novel abstraction of development. Genetic Programming and Evolvable Machines, 8(2), 131-162.

53. Stanley, K. O., Bryant, B. D., \& Miikkulainen, R. (2003). Evolving adaptive neural networks with and without adaptive synapses. In Proceedings of the 2003 IEEE Congress on Evolutionary Computation (CEC-2003).

54. Stanley, K. O., Bryant, B. D., \& Miikkulainen, R. (2005). Real-time neuroevolution in the NERO video game. IEEE Transactions on Evolutionary Computation, 9(6), 653-668.

55. Stanley, K. O., D’Ambrosio, D. B., \& Gauci, J. (2009). A hypercube-based indirect encoding for evolving large-scale neural networks. Artificial Life, 15(2), 185-212.

56. Stanley, K. O., \& Miikkulainen, R. (2002). Evolving neural networks through augmenting topologies. Evolutionary Computation, 10, 99-127.

57. Stanley, K. O., \& Miikkulainen, R. (2003). A taxonomy for artificial embryogeny. Artificial Life, 9(2), 93-130.

58. Stanley, K. O., \& Miikkulainen, R. (2004). Competitive coevolution through evolutionary complexification. Journal of Artificial Intelligence Researh, 21, 63-100.

59. Strobach, P. (1991). Quadtree-structured recursive plane decomposition coding of images. Signal Processing, 39, 1380-1397.

60. Taylor, M. E., Whiteson, S., \& Stone, P. (2006). Comparing evolutionary and temporal difference methods in a reinforcement learning domain. In GECCO 2006: Proceedings of the Genetic and Evolutionary Computation Conference (pp. 1321-1328).

61. Verbancsics, P., \& Stanley, K. O. (2010). Evolving static representations for task transfer. Journal of Machine Learning Research, 99, 1737-1769.

62. Verbancsics, P., \& Stanley, K. O. (2011). Constraining connectivity to encourage modularity in HyperNEAT. In Proceedings of the Genetic and Evolutionary Computation Conference (GECCO 2011).

63. Wagner, G. P., \& Altenberg, L. (1996). Perspective: Complex adaptations and the evolution of evolvability. Evolution, 50(3), 967-976.

64. Watson, J. D., Hopkins, N. H., Roberts, J. W., Steitz, J. A., \& Weiner, A. M. (1987). Molecular biology of the gene (4th ed.). Menlo Park, CA: Benjamin Cummings.

65. Whiteson, S., \& Stone, P. (2006). Evolutionary function approximation for reinforcement learning. Journal of Machine Learning Research, 7, 877-917.

66. Yao, X. (1999). Evolving artificial neural networks. Proceedings of the IEEE, 87(9), 1423-1447.

67. Zigmond, M. J., Bloom, F. E., Landis, S. C., Roberts, J. L., \& Squire, L. R. (Eds.). (1999). Fundamental neurscience. London: Academic Press. 


\section{Appendix I: Experimental Parameters}

All experiments were run with the HyperSharpNEAT Simulator and Experimental Platform v1.0, which builds on a modified version of the public domain SharpNEAT package [21]. The simulator and the ES-HyperNEAT source code can be found at http://eplex.cs.ucf.edu/ESHyperNEAT. Because HyperNEAT differs from the original NEAT only in its set of activation functions, it uses mainly the same parameters [56]. All experiments in this article used the same parameters except as explained below. The size of each population was 300 with $10 \%$ elitism. Sexual offspring $(50 \%)$ did not undergo mutation. Asexual offspring $(50 \%)$ had 0.94 probability of link weight mutation, 0.03 of link addition, and 0.02 of node addition. The NEAT coefficients for determining species similarity were 1.0 for nodes and connections, and 0.1 for weights. Parameter settings are based on standard SharpNEAT defaults and prior reported settings for NEAT [56, 58]. Through preliminary experimentation, they were found to be robust to moderate variation.

The available CPPN activation functions for the dual task and navigation domain were sigmoid, Gaussian, absolute value, and sine. Following Verbancsics and Stanley [62], the activation functions for the retina problem were absolute value, sigmoid, Gaussian, linear, sine, step, and ramp. As in previous work [55], all CPPNs received the length of the queried connection as an additional input. The bandpruning threshold for all ES-HyperNEAT experiments was set to 0.3. Iterated ES-HyperNEAT had an initial and maximum resolution of $8 \times 8$ for the dual task and navigation experiment. The maximum resolution for the retina problem was increased to $32 \times 32$ with a division threshold of 0.5 , reflecting the increased task complexity. The variance and division threshold were set to 0.03 . Finally, the iteration level was 1, which means that ES-HyperNEAT checks for hidden nodes one iteration beyond the first hidden nodes discovered directly from the inputs. 


\section{Appendix 2: ES-HyperNEAT Pseudocode}

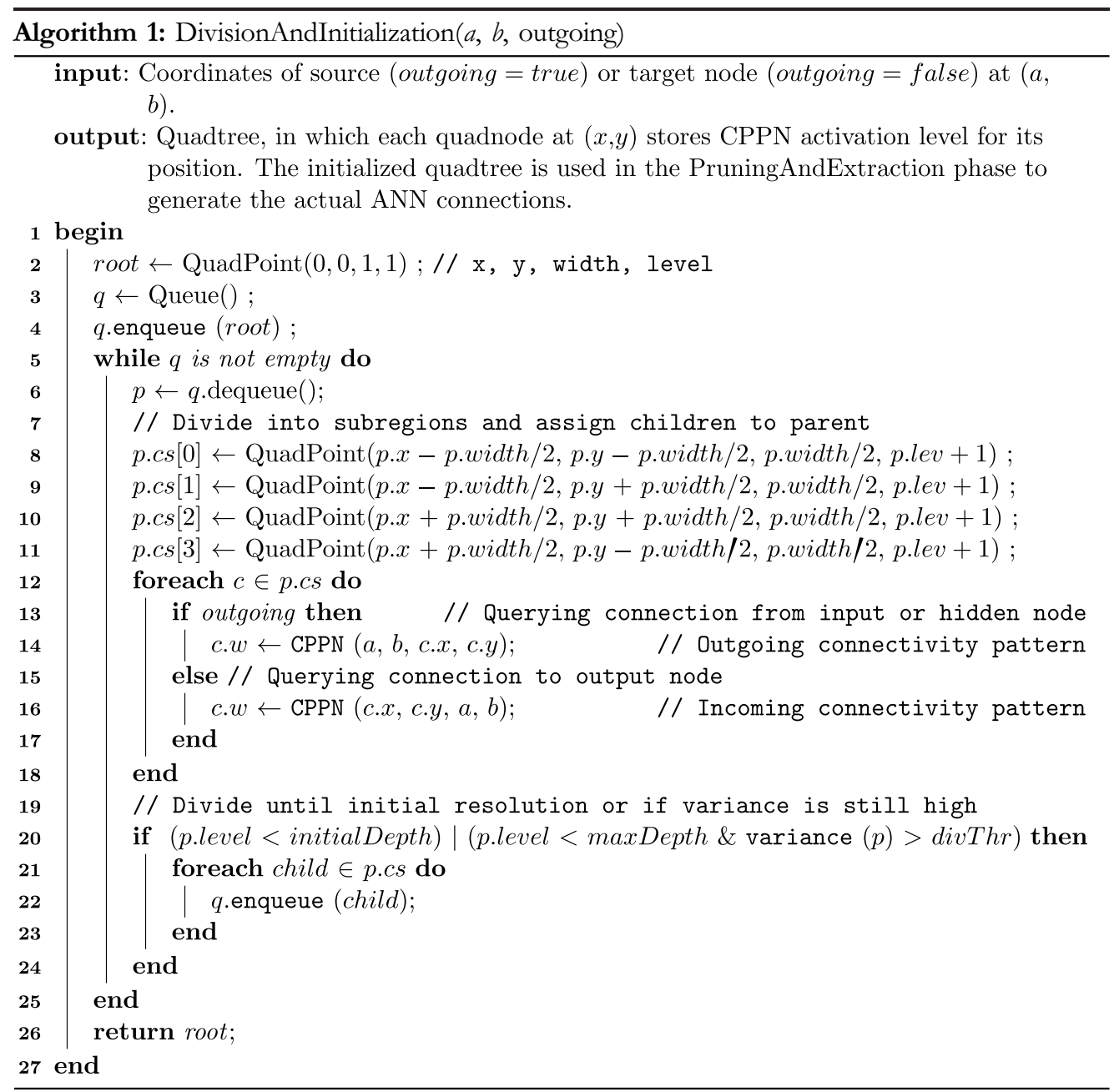




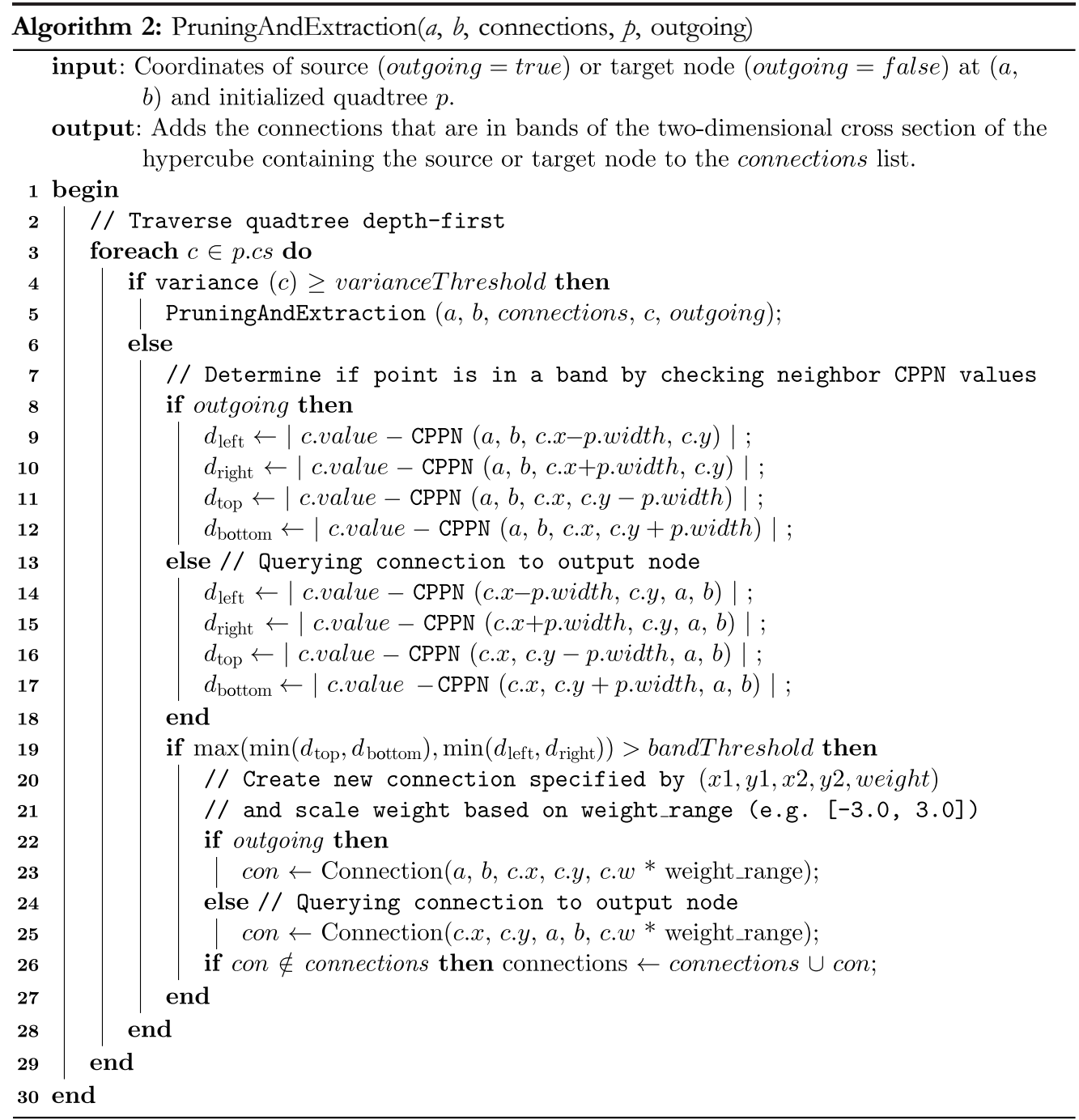




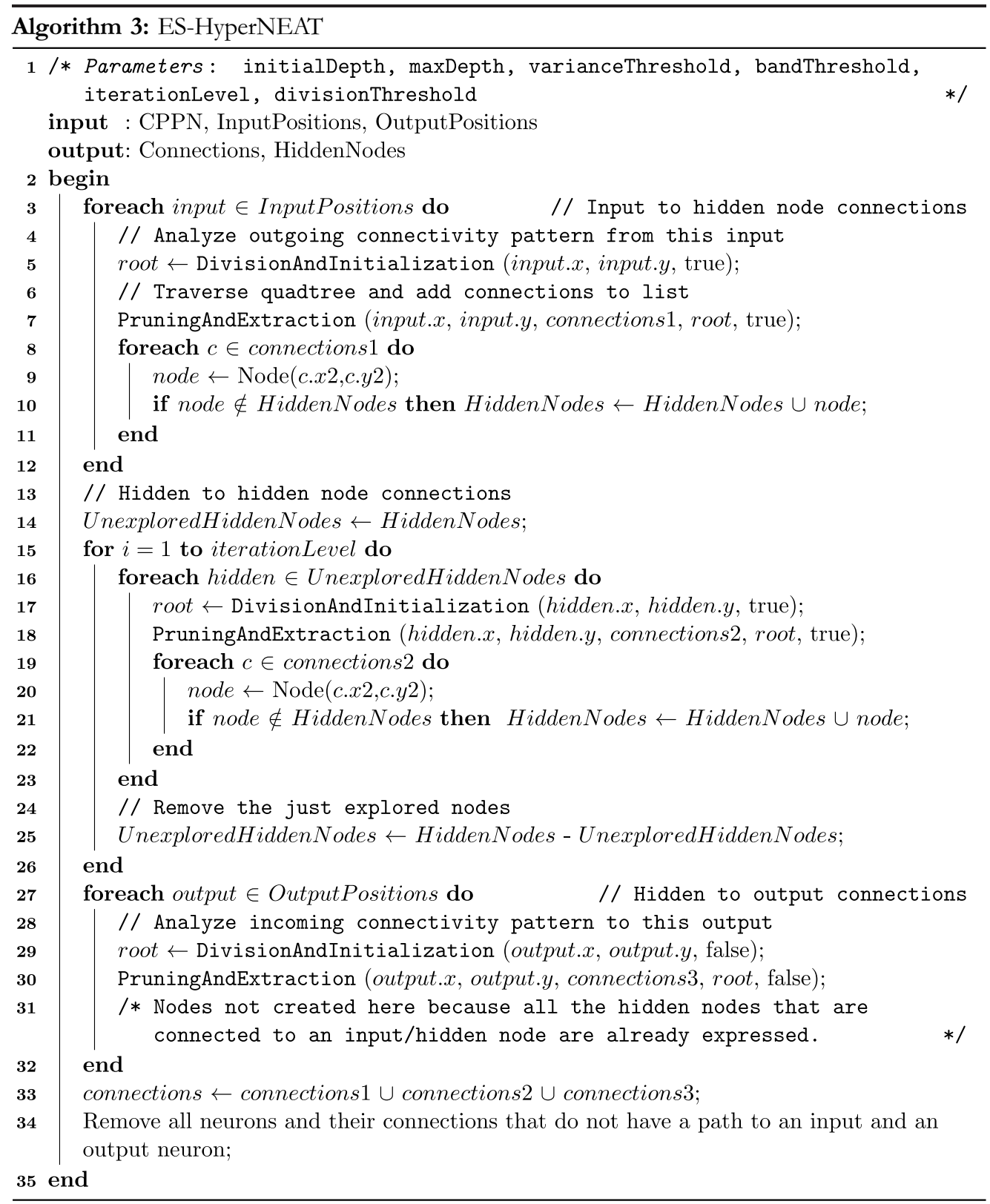


